TRANSACTIONS OF THE

AMERICAN MATHEMATICAL SOCIETY

Volume 355, Number 3, Pages 1079-1108

S 0002-9947(02)03013-1

Article electronically published on November 1, 2002

\title{
DERIVATIONS AND INVARIANT FORMS OF JORDAN AND ALTERNATIVE TORI
}

\author{
ERHARD NEHER AND YOJI YOSHII
}

Dedicated to Holger Petersson

\begin{abstract}
Jordan and alternative tori are the coordinate algebras of extended affine Lie algebras of types $A_{1}$ and $A_{2}$. In this paper we show that the derivation algebra of a Jordan torus is a semidirect product of the ideal of inner derivations and the subalgebra of central derivations. In the course of proving this result, we investigate derivations of the more general class of division graded Jordan and alternative algebras. We also describe invariant forms of these algebras.
\end{abstract}

\section{INTRODUCTION}

This paper provides a detailed description of the derivation algebra Der $J$ of a Jordan torus $J$. In particular, our main result, Theorem 4.11, says that the derivation algebra Der $J$ of a Jordan torus $J$ is a semidirect product,

$$
\text { Der } J=\operatorname{IDer} J \rtimes \operatorname{CDer} J,
$$

of the ideal IDer $J$ of inner derivations and the subalgebra CDer $J$ of central derivations of $J$.

Let us explain the concepts involved in the statement above. We consider (linear) unital Jordan algebras $J$ over a field $F$ of characteristic $\neq 2$. All our algebras are $G$-graded, i.e., $J=\bigoplus_{g \in G} J_{g}, J_{g} J_{g^{\prime}} \subset J_{g+g^{\prime}}$, where $G$ is an abelian group. We call $J$ division graded if every $0 \neq x_{g} \in J_{g}$ is invertible. A division graded $J$ is a Jordan $G$-torus if $\operatorname{dim} J_{g} \leq 1$ for all $g \in G$, and is simply called a Jordan torus if $G=\mathbb{Z}^{n}$. Examples of Jordan tori are the plus algebras of quantum tori (see section 4.2), which recently have gained a lot of interest. Jordan tori have recently been classified in [31].

Perhaps even more important than the decomposition (11) itself is, in our opinion, the concept of central derivations. In general, for a nonassociative $G$-graded algebra $A=\bigoplus_{g \in G} A_{g}$ with centre $Z$ a central derivation $\partial_{\Theta}$ is associated to any group homomorphism $\Theta: G \rightarrow(Z,+)$ by defining $\partial_{\Theta}\left(a_{g}\right)=\Theta(g) a_{g}$ for $a_{g} \in A_{g}$. We denote by CDer $J$ the subalgebra of all central derivations.

We learned the concept of central derivations from the paper [24] by Osborn and Passman where they have been introduced for twisted group algebras and where

Received by the editors April 3, 2001 and, in revised form, January 31, 2002.

2000 Mathematics Subject Classification. Primary 17C10; Secondary 17B60, 17B70, 17C60.

The research of the first author was partially supported by an NSERC (Canada) research grant.

The research of the second author was supported by a Fields Postdoctoral Fellowship (Fall 2000) and a PIMS Postdoctoral Fellowship (2001). 
(11) is proven for these algebras. That the derivation algebra is a semidirect product of the ideal of inner derivations and a subalgebra had been proven before in [4] for quantum tori and in [5] for Cayley tori, see section 4.3. One of the novelties of this paper is that we provide a conceptual description of this subalgebra as the algebra of central derivations.

Although our main motivation is Jordan tori, in the body of the paper we are considering more general algebras, namely Jordan or alternative division graded algebras in $\$ 2$ and the corresponding $G$-tori in $\$ 3$. We do so since the methods and results needed for the Jordan torus case easily generalize to the more general settings, essentially without any extra cost, and since we believe that the corresponding Lie algebras, studied in [32] and [29], are an interesting class of algebras. Therefore, our paper also provides information on Der $T$ for any $G$-torus $T$; see, for example, Theorem 3.2 where a version of (1) is proven for $G$-tori satisfying an additional condition. In $\$ 4$ we then prove (11) for all Jordan tori, by making use of their classification (31]). As already mentioned, (11) has been proven for associative tori in [4] and for nonassociative alternative tori in [5]. Our paper provides a slightly more conceptual proof in the latter case. It is included here since it can be done without any extra cost. Besides the decomposition (1), we also determine the precise structure of $\operatorname{CDer} T$ and IDer $T$. For example, we show that if $G$ is finitely generated and $A$ is division graded, then CDer $A$ is a generalized Witt algebra (Proposition 2.9), more precisely a generalization of the recent generalization of Witt algebras by Doković and Zhao [7].

Our interest in Jordan tori and their derivations comes from the theory of extended affine Lie algebras: it is shown in [31] that the centreless cores of extended affine Lie algebras of type $A_{1}$ are precisely the Tits-Kantor-Koecher algebras of Jordan tori. Moreover, it is proven in the recent paper [2] by Allison and Gao that special classes of Jordan tori enter in the description of the centreless cores of extended affine Lie algebras of reduced non-simply-laced types. In the spirit of the paper 4] by Berman, Gao and Krylyuk on extended affine Lie algebras of type $A_{l}, l \geq 3$ (or [5] for type $\mathrm{A}_{2}$ ) the description (1) is an essential ingredient in the classification of all tame extended affine Lie algebras of type $A_{1}$ and other types. Another ingredient in the construction of extended affine Lie algebras of type $A_{1}$ is invariant forms. It is well known that invariant forms are determined by invariant forms on the corresponding coordinate algebras; see, for example, Koecher's work 17] dealing with Tits-Kantor-Koecher algebras or Benkart's more recent paper [3] for root-graded Lie algebras. We prove in Proposition 4.9 that for Jordan tori there exists, up to scalar multiples, only one nondegenerate graded invariant form. The consequences of our paper for extended affine Lie algebras will be elaborated upon in a sequel to this paper.

This paper generalizes results contained in the second author's Ph.D. thesis written at the University of Ottawa under the supervision of the first author.

\section{BASIC DEFINITIONS AND NOTATION}

In this section we will review some basic concepts. Unless specified otherwise, we will consider nonassociative (= not necessarily associative) algebras over some field $F$ of arbitrary characteristic, denoted ch.F. Our primary interest is in unital alternative and Jordan algebras. Whenever we consider Jordan algebras we will assume that ch. $F \neq 2$, unless explicitly stated otherwise. 
1.1. Preliminaries. Let $A$ be a nonassociative algebra with product written as $a b, a, b \in A$. For $a, b, c \in A$ we define the commutator as $[a, b]=a b-b a=(\operatorname{ad} a)(b)$ and the associator as $(a, b, c)=(a b) c-a(b c)$. The span of all commutators and associators will be denoted $[A, A]$, respectively $(A, A, A)$.

The centre $Z=Z(A)$ of $A$ is defined as $Z(A)=\{z \in A \mid[z, A]=0=(z, A, A)=$ $(A, z, A)\}$. If $A$ is unital, $Z(A)$ is a unital associative commutative subalgebra of $A$ and $A$ is canonically an algebra over $Z$. The interplay between the $F$-algebra and $Z$-algebra structure will be an important feature of this paper. We have $Z(A)=$ $\{z \in A \mid[z, A]=0=(z, A, A)\}$ if $A$ is alternative (but see 2.5) and $Z(A)=\{z \in$ $A \mid(z, A, A)=0\}$ if $A$ is Jordan.

An $F$-linear map $d: A \rightarrow A$ is called a derivation of $A$ if $d(x y)=d(x) y+x d(y)$ for all $x, y \in A$. With the usual commutator, the set of all derivations of $A$ is a Lie algebra denoted $\operatorname{Der}_{F} A$, or Der $A$ if $F$ is clear from the context. Any $d \in \operatorname{Der}_{F} A$ leaves the centre $Z=Z(A)$ invariant and hence $\left.d\right|_{Z} \in \operatorname{Der}_{F} Z$ for $d \in \operatorname{Der}_{F} A$. Moreover, we note that $z d$ is a derivation for any $z \in Z$ and $d \in \operatorname{Der}_{F} A$.

The definition of a derivation of course makes sense if $A$ is an algebra over a unital commutative associative ring, for example a unital $F$-algebra considered as an algebra over its centre $Z$. The Lie algebra of $Z$-linear derivations will be denoted $\operatorname{Der}_{Z} A$. Note that for $d \in \operatorname{Der}_{F} A$, we have $\left.d \in \operatorname{Der}_{Z} A \Leftrightarrow d\right|_{Z}=0$. It is then easily seen that $\operatorname{Der}_{Z} A$ is an ideal of $\operatorname{Der}_{F} A$.

1.2. Central closure. Let $A$ be a unital algebra such that its centre $Z=Z(A)$ does not contain any zero divisors of $A$, i.e., the $Z$-module $A$ is torsion-free. An example of such an algebra is a division $G$-graded algebra where $G$ is an ordered abelian group 2 2.3(d)). Let $\bar{Z}$ be the field of fractions of the integral domain $Z$. The central closure of $A$ is defined as $\bar{A}=\bar{Z} \otimes_{Z} A$, which we consider as an algebra over $\bar{Z}$. We note the following facts 33 :

(i) $x \mapsto 1 \otimes x$ is an embedding of $A$ into $\bar{A}$;

(ii) $\bar{A}$ is central over $\bar{Z}$, i.e., the centre of $\bar{A}$ is $\bar{Z}$;

(iii) $\bar{A}$ is prime (resp. a domain) $\Leftrightarrow A$ is prime (resp. a domain).

Let

$$
\overline{\operatorname{Der}_{Z} A}:=\bar{Z} \otimes_{Z} \operatorname{Der}_{Z} A,
$$

which we consider as a Lie algebra over $\bar{Z}$. There is a canonical map

$$
\overline{\operatorname{Der}_{Z} A} \rightarrow \operatorname{Der}_{\bar{Z}} \bar{A}: u \otimes d \mapsto l_{u} \otimes d,
$$

where $l_{u} \otimes d$ acts on $\bar{Z} \otimes A$ via $\left(l_{u} \otimes d\right)(v \otimes a)=u v \otimes d(a)$. Using the fact that any element in $\overline{\operatorname{Der}_{Z} A}$ has the form $\frac{1}{z} \otimes d$ for some $0 \neq z \in Z$ and $d \in \operatorname{Der}_{Z} A$, it is easily seen that this map is injective. Similarly, $\operatorname{Der}_{Z} A$ embeds into $\overline{\operatorname{Der}_{Z} A}$. After an identification we therefore have

$$
\operatorname{Der}_{Z} A<\overline{\operatorname{Der}_{Z} A}<\operatorname{Der}_{\bar{Z}} \bar{A},
$$

where $<$ indicates a subalgebra. Also, we note:

$$
\text { If } A \text { is a finitely generated algebra over } Z \text {, then } \overline{\operatorname{Der}_{Z} A}=\operatorname{Der}_{\bar{Z}} \bar{A} \text {. }
$$

Indeed, let $\left\{a_{i} \mid 1 \leq i \leq n\right\}$ be a generating set of the $Z$-algebra $A$. For $d \in \operatorname{Der}_{\bar{Z}} \bar{A}$ we have $d\left(1 \otimes a_{i}\right)=\left(1 / z_{i}\right) \otimes b_{i}$ for some $0 \neq z_{i} \in Z$ and $b_{i} \in A$. Thus, putting $z=\prod_{i} z_{i}$, we obtain $z d\left(1 \otimes a_{i}\right) \in 1 \otimes A$. Since $z d$ is $Z$-linear, it follows that $z d(1 \otimes A) \subset 1 \otimes A$. So $\left.z d\right|_{1 \otimes A}=: d_{0} \in \operatorname{Der}_{Z} A$ and $d=\frac{1}{z} d_{0} \in \overline{\operatorname{Der}_{Z} A}$, proving $(1.2 .3)$. 
For an associative algebra $A$, we denote by $A^{+}$the Jordan algebra defined on $A$ with $U$-operator $U_{x} y=x y x$. The bilinear product of $A^{+}$is given by $a . b=\frac{1}{2}(a b+b a)$, where on the right side we have the associative product of $A$. Any subalgebra of $A^{+}$ is then also a Jordan algebra; in particular, this is so for $H(A, *):=\left\{a \in A \mid a^{*}=\right.$ $a\}$, where $*$ is an involution of $A$.

1.3. Lemma. Let $A$ be an associative algebra with involution *. We abbreviate $J=H(A, *)$ and consider $J$ as a Jordan algebra. We assume that

(i) $A$ is a torsion-free $Z(A)$-module

and

(ii) $Z(J)=Z(A) \cap J$.

We let $\bar{A}$, respectively $\bar{J}$, be the central closures of $A$ and $J$.

Then $*$ extends uniquely to an involution $*$ of $\bar{A}$ such that $H(\bar{A}, *) \cong \bar{J}$ over the field of fractions $L$ of $Z(J)$ and $L=H(\bar{A}, *) \cap K$ for $K$ the field of fractions of $Z(A)$.

Proof. The involution $*$ on $\bar{A}$ is given by $\left(\frac{1}{z} \otimes a\right)^{*}=\frac{1}{z^{*}} \otimes a^{*}$ for $0 \neq z \in Z(A)$ and $a \in A$. Since $Z(J) \subset Z(A)$, we have a well-defined $L$-linear map

$$
\varphi: \bar{J}=L \otimes_{Z(J)} J \rightarrow \bar{A}=K \otimes_{Z(A)} A: \frac{1}{z} \otimes_{Z(J)} x \mapsto \frac{1}{z} \otimes_{Z(A)} x .
$$

By construction, its image is contained in $H(\bar{A}, *)$. We claim that, in fact, $\varphi(\bar{J})=$ $H(\bar{A}, *)$, Indeed, let $\frac{1}{z} \otimes_{Z(A)} a \in H(\bar{A}, *)$, with $0 \neq z \in Z(A)$ and $a \in A$. Then $z^{*} a=z a^{*}$ holds in $A$; hence $z a^{*} \in H(A, *)=J$. Since also $z^{*} z \in Z(A) \cap J=Z(J)$, it now follows that $\frac{1}{z} \otimes_{Z(A)} a=\frac{z}{z^{*} z} \otimes_{Z(A)} a^{*}=\frac{1}{z^{*} z} \otimes_{Z(A)} z a^{*} \in \varphi(\bar{J})$.

1.4. Inner derivations. For an associative algebra $A$ and any $x \in A$, the map ad $x: A \rightarrow A: y \mapsto[x, y]$ is a so-called inner derivation. More generally, for an alternative algebra $A$ over a field $F$ with ch. $F \neq 2$, the inner derivations are sums of the derivations $\Delta_{a, b}=R_{[a, b]}-L_{[a, b]}-3\left[L_{a}, R_{b}\right]$, where $L$ and $R$ denote the left, respectively right, multiplication in $A$. For a Jordan algebra $J$, the inner derivations are sums of the derivations $\Delta_{a, b}=\left[L_{a}, L_{b}\right], a, b \in J$ (see, e.g., [14, p. 35 and p. $300])$.

For the classes of algebras considered above, the set of all inner derivations will be denoted by IDer $A$. It is easily seen that IDer $A$ is an ideal of the Lie algebra $\operatorname{Der}_{F} A$, and any inner derivation is $Z$-linear for $Z=Z(A)$ :

$$
\text { IDer } A \triangleleft \operatorname{Der}_{Z} A \text {. }
$$

Moreover, for any $z \in Z(A)$, we have $z \Delta_{a, b}=\Delta_{z a, b}=\Delta_{a, z b}$, from which it easily follows that

$$
\bar{Z} \otimes_{Z} \operatorname{IDer} A=\operatorname{IDer} \bar{A},
$$

where we used the identification (1.2.2).

Example. When $A$ is an associative algebra, any derivation of $A$ is clearly a derivation of the Jordan algebra $A^{+}$. The inner derivations of $A^{+}$are also inner derivations of $A$ since

$$
\left[L_{u}, L_{v}\right]=4 \operatorname{ad}[u, v]
$$

whence over a base field containing $\frac{1}{2}$,

$$
\operatorname{Der} A \subset \operatorname{Der} A^{+} \quad \text { and } \quad[A, A] /(Z(A) \cap[A, A]) \cong \operatorname{IDer} A^{+} \subset \operatorname{IDer} A
$$


(see 2.10.2) and Proposition 3.5 for equality).

1.5. Graded algebras. Let $G$ be an abelian group, written additively, and assume that $A$ is $G$-graded, i.e., $A=\bigoplus_{g \in G} A_{g}$ and $A_{g} A_{h} \subset A_{g+h}$ for all $g, h \in G$. In this paper we will only consider algebras graded by an abelian group, although some results hold for nonabelian groups. For $a \in A$, written in the form $a=\sum_{g \in G} a_{g}$ with $a_{g} \in A_{g}$, the $a_{g}$ will be referred to as the homogeneous components and any $a \in \bigcup_{g \in G} A_{g}$ will be called homogeneous. The support of $A$ is $\operatorname{supp} A:=\{g \in$ $\left.G \mid A_{g} \neq(0)\right\}$. A subspace $B$ of $A$ is graded if $B=\bigoplus_{g \in G}\left(B \cap A_{g}\right)$ in which case we put $B_{g}=B \cap A_{g}$. Examples of graded subspaces are $[A, A],(A, A, A)$, and $Z(A)$. The subgroup of $G$ generated by $\operatorname{supp} Z, Z=Z(A)$, is called the central grading group. If $H$ is this group, then the $Z$-algebra $A$ is $G / H$-graded:

$$
A=\bigoplus_{\bar{g} \in G / H} A_{\bar{g}} \quad \text { for } A_{\bar{g}}=\bigoplus_{g \in \bar{g}} A_{g} .
$$

1.6. Derivations of graded algebras. Let $A=\bigoplus_{g \in G} A_{g}$ be a $G$-graded algebra. For $g \in G$ define

$$
\left(\operatorname{Der}_{F} A\right)_{g}=\left\{d \in \operatorname{Der}_{F} A \mid d\left(A_{h}\right) \subset A_{g+h} \text { for all } h \in G\right\}
$$

and

$$
\operatorname{grDer}_{F} A=\bigoplus_{g \in G}\left(\operatorname{Der}_{F} A\right)_{g} .
$$

It is easily seen that $\operatorname{grDer}_{F} A$ is a subalgebra of $\operatorname{Der}_{F} A$,

$$
\operatorname{grDer}_{F} A<\operatorname{Der}_{F} A \text {, }
$$

called the subalgebra of graded derivations. It is well known that

$$
\operatorname{grDer}_{F} A=\operatorname{Der}_{F} A \text { if } A \text { is a finitely generated } F \text {-algebra }
$$

(see, for example, [11 Prop. 1], where this is proven for Lie algebras). For $Z$ the centre of $A$ we put

$$
\operatorname{grDer}_{Z} A=\bigoplus_{g \in G}\left(\operatorname{Der}_{Z} A\right)_{g}, \text { where }\left(\operatorname{Der}_{Z} A\right)_{g}=\left(\operatorname{Der}_{F} A\right)_{g} \cap \operatorname{Der}_{Z} A .
$$

It is easy to prove that

$$
\operatorname{grDer}_{F} A \cap \operatorname{Der}_{Z} A=\operatorname{grDer}_{Z} A \text {. }
$$

Let $H$ be a subgroup of $G$ (later this will be the grading group of $Z$ ) and put $\bar{G}=G / H$. We then have a $\bar{G}$-grading of $A$ (see section [1.5) and analogously a $\bar{G}$-grading of $\operatorname{grDer}_{F} A$ :

$$
\operatorname{grDer}_{F} A=\bigoplus_{\bar{g} \in \bar{G}}\left(\operatorname{grDer}_{F} A\right)_{\bar{g}}
$$

where

$$
\begin{aligned}
\left(\operatorname{grDer}_{F} A\right)_{\bar{g}} & =\bigoplus_{g^{\prime} \in \bar{g}}\left(\operatorname{grDer}_{F} A\right)_{g^{\prime}} \\
& =\left\{d \in \operatorname{grDer}_{F} A \mid d\left(A_{\bar{a}}\right) \subset A_{\bar{g}+\bar{a}} \text { for all } a \in G\right\} .
\end{aligned}
$$

We will put

$$
\left(\operatorname{grDer}_{F} A\right)_{H}:=\left(\operatorname{grDer}_{F} A\right)_{\overline{0}}=\bigoplus_{h \in H}\left(\operatorname{Der}_{F} A\right)_{h} .
$$


Concerning inner derivations of $G$-graded alternative or Jordan algebras $A$, we have

$$
\text { IDer } A=\bigoplus_{g \in G}(\operatorname{IDer} A)_{g},
$$

where $(\operatorname{IDer} A)_{g}=(\operatorname{Der} A)_{g} \cap \operatorname{IDer} A$. In particular, by (1.4.1),

$$
\text { IDer } A \subset \operatorname{grDer}_{Z} A \text {. }
$$

1.7. Example of a leftsymmetric algebra. The algebra and its graded subalgebra, introduced in this subsection, will be used in section 1.8 to define special types of derivations.

Let $G$ be an abelian group and let $Z=\bigoplus_{g \in G} Z_{g}$ be a commutative associative $G$-graded algebra (later this will be the centre of a nonassociative algebra). We denote by $\operatorname{Hom}_{\mathbb{Z}}(G, Z)$ the $F$-vector space of abelian group homomorphisms from $G$ into the additive group $(Z,+)$. Of course, $\operatorname{Hom}_{\mathbb{Z}}(G, Z)$ also carries a $Z$-module structure which, however, will not be important in the following. We define an $F$-algebra on $\operatorname{Hom}_{\mathbb{Z}}(G, Z)$ by assigning to $\Theta, \Phi \in \operatorname{Hom}_{\mathbb{Z}}(G, Z)$ the product $\Theta * \Phi$, given by

$$
(\Theta * \Phi)(g)=\sum_{h \in G} \Theta(h) \Phi(g)_{h} .
$$

Note that this is well defined since $\Phi(g)_{h} \neq 0$ for finitely many $h \in G$ only. A straightforward calculation shows that the associator with respect to $*$ satisfies

$$
(\Theta, \Phi, \Psi)(g)=-\sum_{h \in G} \Theta(h) \Phi(h) \Psi(g)_{h} .
$$

Therefore, $\left(\operatorname{Hom}_{\mathbb{Z}}(G, Z), *\right)$ if a leftsymmetric algebra, i.e., $(\Theta, \Phi, \Psi)=(\Phi, \Theta, \Psi)$ holds for all $\Theta, \phi, \Psi \in \operatorname{Hom}_{\mathbb{Z}}(G, Z)$. Leftsymmetric algebras, rather their opposite algebras have recently been studied in [10] and [9], where the reader can also find references to previous investigations. In particular, these two papers consider examples of rightsymmetric algebras closely related to the example above.

It is well known and easily checked that for any leftsymmetric algebra, the commutator defines a Lie algebra. It will follow from the results in 1.8, 1.9 and Proposition 2.9 that the Lie algebra $\left(\operatorname{Hom}_{\mathbb{Z}}(G, Z),[\cdot, \cdot]\right)$ can be considered a generalization of the Witt algebra.

We denote by $\operatorname{Hom}_{\mathbb{Z}}(G, Z)_{\text {fin }}$ the $Z$-submodule of all $\Theta$ for which $\Theta(G)$ is contained in the sum of finitely many $Z_{g}$ 's and by $\operatorname{Hom}_{\mathbb{Z}}\left(G, Z_{g}\right)$ the $F$-subspace of those $\Theta \in \operatorname{Hom}_{\mathbb{Z}}(G, Z)$ with $\Theta(G) \subset Z_{g}, g \in G$. It is easily seen that

$$
\begin{aligned}
\operatorname{Hom}_{\mathbb{Z}}(G, Z)_{\text {fin }} & \left.=\bigoplus_{g \in G} \operatorname{Hom}_{\mathbb{Z}}\left(G, Z_{g}\right) \quad \text { (for all } G\right) \\
& =\operatorname{Hom}_{\mathbb{Z}}(G, Z) \quad \text { if } G \text { is finitely generated. }
\end{aligned}
$$

We have $\operatorname{Hom}_{\mathbb{Z}}\left(G, Z_{g_{1}}\right) * \operatorname{Hom}_{\mathbb{Z}}\left(G, Z_{g_{1}}\right) \subset \operatorname{Hom}_{\mathbb{Z}}\left(G, Z_{g_{1}+g_{2}}\right)$ since

$$
\Theta * \Phi=\Theta\left(g_{2}\right) \Phi \quad \text { for } \Theta \in \operatorname{Hom}_{\mathbb{Z}}(G, Z), \Phi \in \operatorname{Hom}_{\mathbb{Z}}\left(G, Z_{g_{2}}\right) .
$$

Hence $\operatorname{Hom}_{\mathbb{Z}}(G, Z)_{\text {fin }}$ is a subalgebra of $\left(\operatorname{Hom}_{\mathbb{Z}}(G, Z), *\right)$, which is $G$-graded by (1.7.3). We note

$$
\operatorname{Hom}(G, Z) * \operatorname{Hom}\left(G, Z_{0}\right)=0
$$


1.8. Central derivations. Let $A$ be a $G$-graded unital algebra over $F$ with centre $Z=\bigoplus_{h \in H} Z_{h}$ and central grading group $H$. For the sake of uniqueness

(1.8.1) we assume in this subsection that $\operatorname{supp} A$ spans $G$ as an abelian group.

In order to apply the results of section 1.7, we will also consider $Z$ as a $G$-graded algebra. Any $\Theta \in \operatorname{Hom}_{\mathbb{Z}}(G, Z)$ gives rise to an $F$-linear derivation $\partial_{\Theta}$ of $A$, defined on $A_{g}$ by

$$
\partial_{\Theta}\left(a_{g}\right)=\Theta(g) a_{g} \quad\left(a_{g} \in A_{g}\right)
$$

and called a central derivation (see [24 section 2], where this concept has been introduced for the case of twisted group algebras). We denote by $\operatorname{CDer}_{F} A$ the space of central derivations. The formula

$$
\left[\partial_{\Theta}, \partial_{\Phi}\right]=\partial_{\Theta * \Phi-\Phi * \Theta}
$$

shows that $\operatorname{CDer}_{F} A$ is a subalgebra of the Lie algebra $\operatorname{Der}_{F} A$ and that

$$
\partial: \operatorname{Hom}_{\mathbb{Z}}(G, Z) \rightarrow \operatorname{CDer}_{F} A
$$

is an epimorphism of Lie algebras. It maps the subalgebra $\operatorname{Hom}_{\mathbb{Z}}(G, Z)_{\text {fin }}$ of $\left(\operatorname{Hom}_{\mathbb{Z}}(G, Z),[\cdot, \cdot]\right)$ onto the graded subalgebra

$$
\left(\operatorname{CDer}_{F} A\right)_{\mathrm{fin}}:=\partial\left(\operatorname{Hom}_{\mathbb{Z}}(G, Z)_{\mathrm{fin}}\right)=\bigoplus_{h \in H}\left(\operatorname{CDer}_{F} A\right)_{h}
$$

of $\operatorname{grDer}_{F} A$, where

$$
\partial\left(\operatorname{Hom}_{\mathbb{Z}}\left(G, Z_{h}\right)\right)=:\left(\operatorname{CDer}_{F} A\right)_{h} \subset\left(\operatorname{Der}_{F} A\right)_{h} .
$$

The derivations in

$$
\mathcal{D}:=\left\{\partial_{\Theta} \mid \Theta \in \operatorname{Hom}_{\mathbb{Z}}\left(G, Z_{0}\right)\right\}
$$

will be called degree derivations. By (1.7.6), $\mathcal{D}$ is always abelian.

1.9. Generalized Witt algebras. Central derivations are related to a generalization of the Witt algebra. This Lie algebra can naturally be defined in the following setting:

(i) $K$ is an associative commutative unital ring, $H$ is an abelian group;

(ii) $Z=\bigoplus_{h \in H} Z_{h}$ is an associative commutative $H$-graded algebra over $K$ (later this will be a twisted group ring $K^{t}[H]$ of $H$ over $K$, or even the group algebra $K[H])$;

(iii) $D$ is a $K$-module; and

(iv) $p: D \times H \rightarrow K:(d, h) \mapsto d(h)$ is a pairing that is $K$-linear in the first variable and additive in the second.

We define

$$
W:=W(Z, D, p):=Z \otimes_{K} D=\bigoplus_{h \in H} Z_{h} \otimes_{K} D
$$

and use the abbreviation $z_{h} d=z_{h} \otimes_{K} d$ for $z_{h} \in Z_{h}, d \in D$. There exists a unique $K$-algebra product $*$ on $W$ such that $\left(z_{h} d_{1}\right) *\left(z_{i} d_{2}\right)=z_{h} d_{1}(i) z_{i} d_{2}$ for homogeneous $z_{h}, z_{i} \in Z, h, i \in H$ and $d_{1}, d_{2} \in D$. It is easily seen that $(W, *)$ is a leftsymmetric algebra. Hence $W$ together with the commutator is a Lie algebra, which we call the generalized Witt algebra associated to $(Z, D, p)$. Thus, the Lie algebra product of $W$ is given by

$$
\left[z_{h} d_{1}, z_{i} d_{2}\right]=z_{h} z_{i}\left(d_{1}(i) d_{2}-d_{2}(h) d_{1}\right) .
$$


For the special case of the group algebra $Z=K[H]$, this Lie algebra has been introduced in [7], where the reader can also find some background information and examples, justifying the terminology. Another special case has been considered in [26] and [28].

In the setting above, the left and right kernel of $p$ are

$$
\begin{aligned}
& D^{H}:=\{d \in D: d(H)=0\} \quad \text { (left kernel), } \\
& H^{D}:=\{h \in H: p(D, h)=0\} \quad \text { (right kernel). }
\end{aligned}
$$

Example. In the setting of section [1.8 we let $K=Z_{0}, H$ the central grading group of $Z=Z(A), D=\operatorname{Hom}_{\mathbb{Z}}\left(G, Z_{0}\right)$ and

$$
p: \operatorname{Hom}_{\mathbb{Z}}\left(G, Z_{0}\right) \times H \rightarrow Z_{0}:(\varphi, h) \mapsto \varphi(h)
$$

the canonical pairing. In this case, $D^{H}=\left\{\varphi \in \operatorname{Hom}_{\mathbb{Z}}\left(G, Z_{0}\right) \mid \varphi(H)=0\right\}$, which is in general nonzero, e.g., for quantum tori. It follows from (1.7.5) that

$$
\mu: W\left(Z, \operatorname{Hom}_{\mathbb{Z}}\left(G, Z_{0}\right), p\right) \rightarrow \operatorname{Hom}_{\mathbb{Z}}(G, Z)_{\text {fin }}: z_{h} \otimes \varphi \mapsto z_{h} \varphi
$$

is a homomorphism of leftsymmetric algebras, hence also for the associated Lie algebras. Note that we now have constructed the following Lie algebra homomorphism:

$$
W\left(Z, \operatorname{Hom}_{\mathbb{Z}}\left(G, Z_{0}\right), p\right) \stackrel{\mu}{\rightarrow} \operatorname{Hom}_{\mathbb{Z}}(G, Z)_{\mathrm{fin}} \stackrel{\partial}{\rightarrow}\left(\operatorname{CDer}_{F} A\right)_{\mathrm{fin}} .
$$

We will show in Proposition 2.9 that for so-called division graded algebras, both maps $\mu$ and $\partial$ are isomorphisms.

1.10. Lemma. Let $W=W(Z, D, p)$ be a generalized Witt algebra as in section 1.9 above.

(a) Any $K$-module $D^{\prime}$ of $D$ gives rise to a subalgebra $Z \otimes_{K} D^{\prime}$ of $W$. Moreover,

$$
Y:=Z \otimes_{K} D^{H}
$$

is an abelian ideal of $W$. If $D=D^{\prime} \oplus D^{H}$ is a direct sum of $K$-modules, e.g., if $K$ is a field, the generalized Witt algebra $W$ is a semidirect product,

$$
W=X \ltimes Y \quad \text { for } X:=Z \otimes_{K} D^{\prime}
$$

of an abelian ideal $Y$ and a subalgebra $X$, which is again a generalized Witt algebra, namely $X=W\left(Z, D^{\prime}, p^{\prime}\right)$, where $p^{\prime}=p \mid D^{\prime} \times H$ has zero left kernel.

(b) If $K$ is a field of characteristic $\neq 2$ and $p \neq 0$, then $W$ is perfect.

Proof. (a) is easy. For (b) we observe that

$$
\left[z_{0} d, z_{h} d\right]=z_{0} z_{h} d(h) d \in K z_{h} d
$$

for $h \in H$ and any $d \in D$. Hence $Z d \subset[W, W]$ for any $d \notin D^{H}$. But for $d \in D^{H}$ and any $d_{1} \in D$, we have $\left[z_{h} d_{1}, z_{i} d\right]=z_{h} z_{i} d_{1}(i) d$. Since $p \neq 0$, there exist $d_{1}$ and $i \in H$ such that $d_{1}(i) \neq 0$ and so $z_{k} d \in[W, W]$ for any $k \in H$.

Remark. For the special case that $K$ is a field of characteristic zero and $Z$ is the group algebra of $H$, the structure of $W$ is determined in 16. In particular, a more precise version of (1.10.2) is shown there: $W$ is a semidirect product of the abelian ideal $Y$ and a simple subalgebra $X$, which is again a generalized Witt algebra. We will prove an analogue in our setting in Theorem 4.12 . 
1.11. Example: $H(A, *)$ for $*$ of second kind. Let $A=\bigoplus_{g \in G} A_{g}$ be a unital associative $G$-graded algebra over a field $E$ of ch. $E \neq 2$ and suppose $*$ is a graded involution of $A$, i.e., $A_{g}^{*}=A_{g}$ for all $g \in G$, which is of second kind in the sense that $E .1=(E .1)^{*} \not \subset H(A, *)$. For simpler notation let us put

$$
H:=H(A, *) \quad \text { and } \quad S:=S(A, *)=\left\{a \in A \mid a^{*}=-a\right\} .
$$

We identify $E=E$.1. With $F=E \cap H$ we have that $E / F$ is a Galois extension of degree 2 ; so $E=F[s]$ for some $s=-s^{*} \in E$. Then

$$
A=H \oplus s H, \text { where } s H=S
$$

as $G$-graded spaces. It follows that the supports of the associative algebra $A$ and the Jordan algebras $A^{+}$and $H$ all coincide:

$$
\operatorname{supp} A=\operatorname{supp} A^{+}=\operatorname{supp} H .
$$

Since $[a, b]^{*}=-\left[a^{*}, b^{*}\right]$ and the Lie product $[\cdot, \cdot]$ is $E$-linear, (1.11.1) implies

$$
[A, A]=[H, H] \oplus[H, S] \text { with }[A, A] \cap S=[H, H]=[S, S]
$$

and $[A, A] \cap H=[H, S]=s[H, H]$. We have the general formula

$$
A \ni[x,[y, z]]=4(y, x, z) \in A^{+},
$$

where the left and right side are calculated in $A$ and $A^{+}$, respectively. Hence the centres of the Jordan algebras $A^{+}$and $H$ are given by

$$
\begin{gathered}
Z\left(A^{+}\right)=\{z \in A \mid[A,[A, z]]=0\}, \\
Z(H)=\{z \in H \mid[H,[H, z]]=0\}=Z\left(A^{+}\right) \cap H,
\end{gathered}
$$

where the second equality in (1.11.5) follows from the $E$-linearity of $[\cdot, \cdot]$.

Let us now consider derivations. The involution $*$ induces a Lie algebra automorphism $\operatorname{Der}_{E} A^{+} \rightarrow \operatorname{Der}_{E} A^{+}: d \mapsto d^{*}$ of order 2 , where $d^{*}$ is defined by $d^{*}(a)=d\left(a^{*}\right)^{*}$. Hence, with obvious notation,

$$
\operatorname{Der}_{E} A^{+}=H\left(\operatorname{Der}_{E} A^{+}, *\right) \oplus S\left(\operatorname{Der}_{E} A^{+}, *\right) .
$$

Any $d \in H\left(\operatorname{Der}_{E} A^{+}, *\right)$ leaves $H$ invariant. Hence we have a well-defined map

$$
\Psi: H\left(\operatorname{Der}_{E} A^{+}, *\right) \rightarrow \operatorname{Der}_{F} H:\left.d \mapsto d\right|_{H} .
$$

In fact, $\Psi$ is an isomorphism. Injectivity of $\Psi$ follows from 1.11.1) and for surjectivity one notes that any $d \in \operatorname{Der}_{F} H$ extends to a $\tilde{d} \in \operatorname{Der}_{E} A^{+}$with $\tilde{d}^{*}=\tilde{d}$ by defining $\tilde{d}\left(h+s h^{\prime}\right)=d(h)+s d\left(h^{\prime}\right)$ for $h, h^{\prime} \in H$. In the following we will identify $\operatorname{Der}_{F} H=H\left(\operatorname{Der}_{E} A^{+}, *\right)$ via $\Psi$. We then have

$$
\operatorname{Der}_{E} A^{+}=\operatorname{Der}_{F} H \oplus s \operatorname{Der}_{F} H,
$$

where $s \operatorname{Der}_{F} H=S\left(\operatorname{Der}_{E} A^{+}, *\right)$. By construction, $\Psi$ preserves the homogeneous spaces. Hence $\left(\operatorname{Der}_{F} H\right)_{g}$ is the fixed point space of the automorphism $*$, and because of (1.11.6) we then have

$$
\operatorname{dim}_{E}\left(\operatorname{Der}_{E} A^{+}\right)_{g}=\operatorname{dim}_{F}\left(\operatorname{Der}_{F} H\right)_{g}
$$

for all $g \in G$. For inner derivations, (11.4.3) implies

$$
\operatorname{IDer} A^{+}=\operatorname{ad}[A, A]=\operatorname{ad}[H, H] \oplus \operatorname{ad}[H, S]=\operatorname{ad}[H, H] \oplus s \operatorname{ad}[H, H],
$$


where the second equality follows from (1.11.3) and $(\operatorname{ad} a)^{*}=\operatorname{ad}\left(-a^{*}\right)$. Hence again by (1.4.3),

$$
\text { IDer } H=\operatorname{ad}[H, H]=\operatorname{IDer} A^{+} \cap \operatorname{Der}_{F} H
$$

so that (1.11.7) also holds for inner derivations. For a central derivation $\partial_{\Theta}$ of $A^{+}$we have $\left(\partial_{\Theta}\right)^{*}=\partial_{\Theta^{*}}$ for $\Theta^{*}(g)=(\Theta(g))^{*}$. The map $\Theta \mapsto \Theta^{*}$ leaves $\operatorname{Hom}_{\mathbb{Z}}\left(G, Z\left(A^{+}\right)\right)$invariant. By (1.11.5), any $\Theta$ with $\Theta^{*}=\Theta$ maps $G$ into $Z(H)$ and hence $\operatorname{Hom}_{\mathbb{Z}}\left(G, Z\left(A^{+}\right)\right)=\operatorname{Hom}_{\mathbb{Z}}(G, Z(H)) \oplus s \operatorname{Hom}_{\mathbb{Z}}(G, Z(H))$, which implies

$$
\operatorname{CDer}_{E} A^{+}=\operatorname{CDer}_{F} H \oplus s \operatorname{CDer}_{F} H \text {. }
$$

Therefore, $\operatorname{CDer}_{F} H=\operatorname{CDer}_{E} A^{+} \cap \operatorname{Der}_{F} H$ and (1.11.7) also holds for central derivations.

1.12. Graded invariant forms. A graded invariant form on a $G$-graded $F$-algebra $A=\bigoplus_{g \in G} A_{g}$ is a symmetric bilinear form $(\cdot \mid \cdot): A \times A \rightarrow F$ satisfying

(i) $\left(A_{g} \mid A_{h}\right)=0$ for $g+h \neq 0$,

and

(ii) $(a b \mid c)=(a \mid b c)$ for all $a, b, c \in A$.

The $F$-vector space of all graded invariant forms on $A$ will be denoted $\mathcal{G} \mathcal{I} \mathcal{F}(A)$. It is described in the following Lemma 1.13 whose proof will be left to the reader.

We will say that a graded invariant form $(\cdot \cdot \cdot)$ is nondegenerate if its radical $\operatorname{rad}(\cdot \mid \cdot)=\{a \in A \mid(a \mid A)=0\}$ vanishes. Clearly, $\operatorname{rad}(\cdot \mid \cdot)$ is a graded ideal. Hence, if $A$ is graded-simple, i.e., $A$ does not have nonzero graded ideals, then any nonzero graded invariant form is nondegenerate.

1.13. Lemma (graded invariant forms). Let $A$ be a G-graded algebra over a field $F$, put $\operatorname{GIF}(A)=A_{0} /\left([A, A]_{0}+(A, A, A)_{0}\right)$ and denote by $\pi$ the map $A \rightarrow \operatorname{GIF}(A): a=$ $\sum_{g \in G} a_{g} \mapsto \overline{a_{0}}$, where $\overline{a_{0}}$ is the image of $a_{0} \in A_{0}$ under the canonical projection of $A_{0}$ onto $\operatorname{GIF}(A)$. Then every linear form $f \in \operatorname{GIF}(A)^{*}=\operatorname{Hom}_{F}(\operatorname{GIF}(A), F)$ gives rise to a graded invariant form $(\cdot \mid \cdot)_{f}$ defined by $(a \mid b)_{f}=f(\pi(a b))$. The map

$$
\operatorname{GIF}(A)^{*} \rightarrow \mathcal{G} \mathcal{I F}(A): f \mapsto(\cdot \mid \cdot)_{f}
$$

is F-linear, and is an isomorphism if $A$ is unital. In particular,

$$
[A, A]_{0}=0=(A, A, A)_{0} \text { and } A \text { unital } \Rightarrow \mathcal{G I F}(A) \cong A_{0}^{*} .
$$

1.14. Skew derivations. Let again $A$ be a $G$-graded $F$-algebra and let $(\cdot \mid \cdot)$ be a graded invariant form. A derivation $D$ is called a skew derivation if $(d(a) \mid b)+$ $(a \mid d(b))=0$ for all $a, b \in A$. The set of all skew derivations is a subalgebra of $\operatorname{Der}_{F} A$, denoted $\operatorname{SDer}_{F} A$. It is straightforward to check that

$\operatorname{IDer}_{F} A \subset \operatorname{SDer}_{F} A$ for $A$ alternative or Jordan, ch. $F \neq 2$.

It is also easily seen that a graded derivation is skew if and only if every homogeneous component is skew. Thus we have

$$
\operatorname{grSDer}_{F} A:=\operatorname{grDer}_{F} A \cap \operatorname{SDer}_{F} A=\bigoplus_{g \in G}\left(\operatorname{SDer}_{F} A\right)_{g},
$$


where $\left(\operatorname{SDer}_{F} A\right)_{g}:=\operatorname{SDer}_{F} A \cap\left(\operatorname{Der}_{F} A\right)_{g}$. Assume $A$ is unital and let $Z=$ $\bigoplus_{h \in H} Z_{h}$ be the centre of $A$. Then for any $h \in H$,

$$
\begin{gathered}
\operatorname{CDer}_{F} A \cap\left(\operatorname{SDer}_{F} A\right)_{h}=\left\{\partial_{\Theta} \mid \Theta \in \operatorname{Hom}_{\mathbb{Z}}\left(G, Z_{h}\right), \Theta(h)=0\right\} \\
\text { if }(\cdot \mid \cdot) \text { is nondegenerate and } A \text { is unital. }
\end{gathered}
$$

Indeed, if $\Theta \in \operatorname{Hom}_{\mathbb{Z}}\left(G, Z_{h}\right)$, then $\partial_{\Theta} \in \operatorname{SDer}_{F} A$ if and only if for all $g \in G$ and $a_{g} \in A_{g}, b_{-g-h} \in A_{-g-h}$ we have $0=\left(\Theta(g) a_{g} \mid b_{-g-h}\right)+\left(a_{g} \mid \Theta(-g-h) b_{-g-h}\right)=$ $-\left(a_{b} g_{-g-h} \mid \Theta(h)\right)$. Since $A$ is unital and $(\cdot \mid \cdot)$ is nondegenerate, this is equivalent to $\Theta(h)=0$.

\section{Division GRAded ALGEBras}

We specialize the setup of the previous section 1 and consider a class of unital $G$-graded algebras, which we assume to be alternative or Jordan. In the latter case, we will suppose that ch. $F \neq 2$, unless explicitly stated otherwise. We begin with a result on reflection spaces, which naturally arise as the support of division graded algebras.

2.1. Reflection spaces. A set $M$ together with an operation $M \times M \rightarrow M$ : $(m, n) \mapsto m \cdot n$ is a reflection space in the sense of Loos ([19]) if it satisfies the three axioms $m \cdot m=m, m \cdot(m \cdot n)=n$ and $m \cdot(n \cdot p)=(m \cdot n) \cdot(m \cdot p)$ for all $m, n, p \in M$. A homomorphism between reflection spaces is a map preserving the products.

Now let $G$ be an abelian group. With respect to the operation $g \cdot h=2 g-h$, the set $G$ becomes a reflection space. It has a distinguished base point, the zero element 0 of $G$. The following formulas hold in $G$ :

$$
0 \cdot g=-g
$$

and

$$
\begin{gathered}
g_{1} \cdot\left(g_{2} \cdot\left(\cdots\left(g_{m-1} \cdot g_{m}\right) \cdots\right)\right) \\
=2\left(g_{1}-g_{2}+g_{3}-\cdots+(-1)^{m} g_{m-1}\right)+(-1)^{m+1} g_{m} .
\end{gathered}
$$

For a subset $S$ of $G$, we denote by $\langle S\rangle$ the subgroup spanned by $S$. The following conditions are equivalent for $S \subset G$ :

(i) $0 \in S$ and $S \cdot S \subset S$;

(ii) $2\langle S\rangle \subset S$ and $2\langle S\rangle+S \subset S$;

(iii) $S$ is a union of cosets modulo $2\langle S\rangle$ including the trivial coset $2\langle S\rangle$.

Indeed, if (i) holds we have $-S=S$ by (2.1.1) and hence every $g \in\langle S\rangle$ can be written in the form $g=s_{1}-s_{2}+s_{3}-\cdots+(-1)^{n+1} s_{n}$ for suitable $s_{i} \in S$ and $n \geq 1$. For arbitrary $s \in S$, we then obtain $2 g+s \in S$ from (2.1.2); thus, $2\langle S\rangle+S \subset S$ and, in particular, $2\langle S\rangle \subset S$ since $0 \in S$. The implications (ii) $\Rightarrow$ (iii) and (iii) $\Rightarrow$ (i) are immediate.

A subset $S$ satisfying the equivalent conditions (i)-(iii) above will be called a reflection subspace of $G$. The special case of $S \subset G=\left(\mathbb{Z}^{n},+\right)$ and $\langle S\rangle=\mathbb{Z}^{n}$ has been treated in [1, Ch. II, §1] where $S$ was called a semilattice. The description of reflection subspaces above is, in fact, a straightforward generalization of [1, Ch. II, Prop. 1.4 and Remark 1.6].

2.2. Proposition. Let $S \subset G$ be a reflection subspace.

(a) If $G$ is finitely generated as an abelian group, then $S$ is finitely generated as a reflection space. 
(b) Let $H$ be an abelian group without 2-torsion. Then any homomorphism $\varphi: S \rightarrow H$ of reflection spaces preserving the base points uniquely extends to a group homomorphism $\Phi:\langle S\rangle \rightarrow H$.

Proof. (a) Since a subgroup of a finitely generated group is again finitely generated, we may assume $\langle S\rangle=G$. The quotient $G / 2 G$ is a finitely spanned $\mathbb{Z} / 2 \mathbb{Z}$-vector space, hence finite. It therefore follows from section 2.1 that $S$ is the union of a finite number of cosets modulo $2 G$, say $\tau_{i}+2 G, 0 \leq i \leq n$, where we can assume $\tau_{0}=0$.

We next note that $S$ contains a finite generating set of the abelian group $G$. Indeed, by assumption, there exist finitely many elements $x_{1}, x_{2}, \ldots, x_{m} \in G$ spanning $G$. But since $G=\langle S\rangle$ and $S=-S$, any $x_{i}$ is a finite sum of elements of $S$, say $x_{i}=\sum_{j} \sigma_{i j}$ with $\sigma_{i j} \in S$. Hence the collection of all $\left\{\sigma_{i j}\right\}$ is a finite generating set of $G$ contained in $S$.

Let $X=\left\{\sigma_{i j}\right\} \cup\left\{\tau_{0}, \ldots, \tau_{n}\right\}$ and denote by $S^{\prime}$ the reflection space generated by $X$. By 2.1(ii) applied to $S^{\prime}$, we have $\mathbb{Z} y \subset S^{\prime}$ for any $y \in S^{\prime}$. Hence, by choosing appropriate $g_{i}$ in $\bigcup_{i} \mathbb{Z} \sigma_{i j} \cup\left\{ \pm \tau_{i}\right\} \subset S^{\prime}$, (2.1.2) shows that $2 g+\tau_{i} \in S^{\prime}$ for every $g \in G$ and $\tau_{i}, 0 \leq i \leq n$, proving $S^{\prime}=S$.

(b) We may again assume $\langle S\rangle=G$. Since $\varphi(0)=0$ by assumption, we have $\varphi(-s)=\varphi(0 \cdot s)=0 \cdot \varphi(s)=-\varphi(s)$ for every $s \in S$ and also $\varphi(2 s)=\varphi(s \cdot 0)=2 \varphi(s)$. Now for any $g \in G, s \in S$ we obtain $\varphi(2 s-2 g)=\varphi(s \cdot 2 g)=\varphi(s) \cdot \varphi(2 g)=$ $2 \varphi(s)-\varphi(2 g)$. Hence, if $\varphi(2 g) \in 2 H$, so is $\varphi(2(s-g))$. Since any $g \in G$ can be written in the form $g=s_{1}-s_{2}+s_{3}-\cdots+(-1)^{n+1} s_{n}$, it follows by induction on $n$ that $\varphi(2 g) \in 2 H$. We define $\Phi: G \rightarrow H$ by $\Phi(g)=\frac{1}{2} \varphi(2 g)$. Then $\Phi$ extends $\varphi$, and for $s \in S$ and $g \in G$ we have

$$
\begin{aligned}
2 \Phi(s+g) & =\varphi(2 s+2 g)=\varphi(s \cdot(-2 g)) \\
& =\varphi(s) \cdot \varphi(-2 g)=2 \varphi(s)+\varphi(2 g)=2(\Phi(s)+\Phi(g)),
\end{aligned}
$$

whence $\Phi(s+g)=\Phi(s)+\Phi(g)$. Since any $g \in G$ is a finite sum of elements in $S$, this implies, by induction, that $\Phi$ is a group homomorphism.

2.3. Strongly graded and division graded algebras. From now on we will assume that $A$ is a unital alternative or a unital Jordan algebra over $F$. Unless specified otherwise, we will assume that ch.F $\neq 2$ if $A$ is Jordan.

We will say that a $G$-graded algebra $A$ is strongly graded or that $A$ has a $G$ grading of strong type if $A_{g} A_{h}=A_{g+h}$ for all $g, h \in G$. The algebra $A$ is called a division $G$-graded algebra if all nonzero homogeneous elements are invertible. We list some known properties of division graded algebras.

(a) In any alternative algebra, an element $a$ is invertible if and only if the left multiplication $L_{a}$ is invertible, and in this case $\left(L_{a}\right)^{-1}=L_{a^{-1}}$. Moreover, if $a, b$ are invertible, then so is their product $a b$, and then $(a b)^{-1}=b^{-1} a^{-1}$. This easily implies that a division $G$-graded alternative algebra is strongly graded and that its support is a subgroup of $G$ ([29] or [23, I.4.5] for the case of associative algebras).

A division $G$-graded associative algebra, sometimes also called a graded division ring [23], is a crossed product algebra $D * G$ of $G$ over an associative division algebra $D$, and conversely [25] Ch. I $\S 2]$. The classification of division $\mathbb{Z}^{n}$-graded alternative algebras is given in [29].

(b) For a Jordan algebra $A$, we denote by $U_{a}$ the $U$-operator of a Jordan algebra, i.e., $U_{a} b=2 a(a b)-a^{2} b$. Since $A$ is supposed to have a unit element 1 , one can 
recover the bilinear product from the $U$-operator in view of the formula $2 a b=$ $\left(U_{a+b}-U_{a}-U_{b}\right) 1$. An element $a \in A$ is invertible if and only if $U_{a}$ is invertible.

Let $A$ be a division graded Jordan algebra and denote by $S=\operatorname{supp} A$ its support. Then $U_{A_{g}} A_{h}=A_{2 g+h}$ for all $g, h \in S$. Hence $S$ is a reflection subspace of $G$. We note that, in general, $S$ is not a subgroup of $G$ (see, for example, section 4.4).

(c) (29] or 23 I.4.2] for associative algebras) For a totally ordered abelian group $G$, e.g. $G=\mathbb{Z}^{n}$, any division $G$-graded algebra $A$ is a domain in the sense that $x y=0 \Rightarrow x=0$ or $y=0$ if $A$ is alternative, and $U_{x} y=0 \Rightarrow x=0$ or $y=0$ if $A$ is Jordan. We recall that any domain is strongly prime, meaning nondegenerate and prime.

(d) The centre $Z$ of a division $G$-graded algebra $A$ is again division graded. In particular, $Z_{0}$ is a field and $Z=Z_{0}^{t}[H]$ is a twisted group algebra where $H$ is the central grading group. Moreover, $A$ is a division $G$-graded algebra over $Z_{0}$. If $G$ is totally ordered, $Z$ is an integral domain.

(e) Since $A$ is graded-simple, any nonzero graded invariant form on $A$ is nondegenerate. For the existence of such forms see section 1.13, Proposition 3.3, and Proposition 4.9.

We next present some preliminary results on division graded algebras.

2.4. Lemma. Let $A$ be a division G-graded algebra such that

(i) $G$ is finitely generated and

(ii) $\operatorname{dim}_{F} A_{g}<\infty$ for all $g \in G$.

Then $A$ is finitely generated as an algebra.

Proof. Let $X \subset S=\operatorname{supp} A$ be a finite generating set of the reflection space $S$ and let $Y$ be the union of vector space bases of all $A_{g}, g \in X$. The subalgebra $A^{\prime}$ generated by $Y$ is graded and has support $S$, in view of section 2.1 and (a), (b) of section 2.3 By induction on the number of generators needed to express $s \in S$ as a product of factors from $X$, one shows $A_{s}^{\prime}=A_{s}$ for all $s \in S$, hence $A^{\prime}=A$.

2.5. Lemma. If $A$ is an alternative division graded algebra, then

$$
Z(A)=\{a \in A \mid[a, A]=0\} .
$$

Proof. It suffices to show that any homogeneous $a \in A$ satisfying $[a, A]=0$ lies in the centre of $A$. It is proven in McCrimmon's unpublished book [20, III, Lemma 4.1] that $(a, A, A)^{2}=0$ for all $a \in A$ with $[a, A]=0$. In particular, for homogeneous $b, c \in A$, the equation $(a, b, c)^{2}=0$ forces $(a, b, c)=0$. Since [20] is not published, we mention that, for the special case of a prime alternative algebra over a field of characteristic $\neq 3$, the lemma follows from results in [33. Indeed, for the proof of (2.5.1) we may assume that $A$ is not associative. Then $A$ is nondegenerate ([33. 9, Thm. 5]), hence its central closure $\bar{A}$ is a simple alternative algebra (proof of [33, 9, Thm. 9]) and (2.5.1) holds for $\bar{A}$ by [33, 7, Cor. of Lemma 7 and 7, Cor. 1 of Lemma 1]. In particular, any $a \in A$ that commutes with $A$ and hence with $\bar{A}$ associates with everything of $A \subset \bar{A}$ proving (2.5.1).

2.6. Lemma. Let $A$ be a division $G$-graded algebra whose central closure $\bar{A}$ exists (for example, this holds by 2.3(c) if $G$ is ordered) and has only inner derivations. Then IDer $A=\operatorname{grDer}_{Z} A$. 


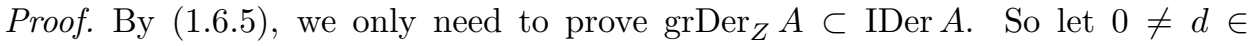
$\operatorname{grDer}_{Z} A$. We may assume that $d$ is homogeneous. By (1.2.2), we have the derivation $\tilde{d}=\operatorname{Id} \otimes d$ of $\bar{A}$ which, by assumption and (1.4.2), has the form $\tilde{d}=\frac{1}{z} \Delta$ for some $0 \neq z \in Z$ and some $\Delta \in \operatorname{IDer} A$. Hence $z \tilde{d}=\Delta$ on $\bar{A}$ and so, by restriction, $z d=\Delta$ on $A$. Considering the homogeneous components of this equation, we obtain a nonzero homogeneous $z^{\prime} \in Z$ and a homogeneous $\Delta^{\prime} \in$ IDer $A$ such that $z^{\prime} d=\Delta^{\prime}$. Since $z^{\prime}$ is invertible, it follows that $d=\frac{1}{z^{\prime}} \Delta^{\prime} \in \operatorname{IDer} A$.

2.7. Graded modules. Let $A=\bigoplus_{g \in G} A$ be an associative division $G$-graded algebra over $F$. Suppose that the group $G$ acts on a set $S$. A left $A$-module $M$ is called a graded $A$-module of type $S$ if $M=\bigoplus_{s \in S} M_{s}$ and $A_{g} M_{s} \subset M_{g . s}$ for each $g \in G$ and $s \in S$. In this case, we let $\bar{s}:=G . s \in S / G$ be the $G$-orbit of $s$ and put $M_{\bar{s}}:=\bigoplus_{g \in G} M_{g . s}$. Obviously, $M=\bigoplus_{\bar{s} \in S / G} M_{\bar{s}}$.

The following lemma is easy to prove. For related results see, for example, [6] Thm. 3], [23, I.3.4] and [31, Lemma 3.6].

2.8. Lemma. Let $A=\bigoplus_{g \in G} A_{g}$ be an associative division G-graded algebra and $M=\bigoplus_{s \in S} M_{s}$ a graded A-module of type $S$. Then:

(a) $M_{g . s}=a_{g} M_{s}=A_{g} M_{s}$ for all $g \in G, s \in S$ and any $0 \neq a_{g} \in A_{g}$. Hence $A M_{s}=M_{\bar{s}}$.

(b) Suppose that $G$ acts freely on $S$, i.e., g.s $=s$ for some $g \in G$ and $s \in S$ implies $g=0$. Then $M_{\bar{s}}$ is a free A-module; namely, any $A_{0}$-basis of the $A_{0}$ vector space $M_{s}$ is also an A-basis of $M_{\bar{s}}$. Hence $\operatorname{rank}_{A} M_{\bar{s}}=\operatorname{dim}_{A_{0}} M_{s^{\prime}}$ for all $s^{\prime} \in \bar{s}$. Moreover, $M$ is a free A-module, there exists an A-basis of $M$ consisting of homogeneous elements and $M \cong A \otimes_{A_{0}} N$ for a suitable $A_{0}$-module $N$.

(c) Suppose that $G$ and $S$ are totally ordered and that the action preserves the order, i.e., $g<g^{\prime}$ and $s<s^{\prime}$ implies g.s $<g^{\prime} . s^{\prime}$. Then any graded submodule $N$ of $M$ is saturated in the sense that $N=\{m \in M \mid a m \in N$ for some $0 \neq a \in A\}$. Hence, for any multiplicatively closed subset $B$ of $A$ we have $B^{-1} M=B^{-1} N \Leftrightarrow$ $M=N$.

2.9. Proposition. Let $A$ be a division graded algebra with $G=\langle\operatorname{supp} A\rangle$, and $Z=$ $\bigoplus_{h \in H} Z_{h}$ the centre of $A$ with central grading group $H$. We choose $0 \neq z_{h} \in Z_{h}$ for all $h \in H$. Recall that $\mathcal{D}$ denotes the degree derivations as defined in (1.8.5). Then the maps $\mu$ and $\partial$ of (1.9.4),

$$
W\left(Z, \operatorname{Hom}_{\mathbb{Z}}\left(G, Z_{0}\right), p\right) \stackrel{\mu}{\rightarrow} \operatorname{Hom}_{\mathbb{Z}}(G, Z)_{\mathrm{fin}} \stackrel{\partial}{\rightarrow}\left(\operatorname{CDer}_{F} A\right)_{\mathrm{fin}}
$$

are isomorphisms of Lie algebras. Moreover,

$$
\begin{aligned}
\operatorname{Hom}_{\mathbb{Z}}(G, Z)_{\mathrm{fin}} & =\bigoplus_{h \in H} z_{h} \operatorname{Hom}_{\mathbb{Z}}\left(G, Z_{0}\right) \cong Z \otimes_{Z_{0}} \operatorname{Hom}_{\mathbb{Z}}\left(G, Z_{0}\right), \\
\left(\operatorname{CDer}_{F} A\right)_{\mathrm{fin}} & =\bigoplus_{h \in H} z_{h} \mathcal{D}=Z \otimes_{Z_{0}} \mathcal{D}, \text { and } \\
\operatorname{SDer}_{F} A \cap\left(\operatorname{CDer}_{F} A\right)_{\mathrm{fin}} & =\bigoplus_{h \in H} z_{h}\left\{\partial_{\Theta} \in \mathcal{D}: \Theta(h)=0\right\}
\end{aligned}
$$

with respect to any nondegenerate graded invariant form on $A$.

Proof. Equations (2.9.1) and (2.9.2) are immediate from Lemma 2.8, while (2.9.3) follows from (1.14.3). In particular, (2.9.1) implies that $\mu$ is an isomorphism. To show that $\partial$ is an isomorphism it suffices in view of (1.8.3) to prove injectivity. 
Assume therefore that $z_{h} \partial_{\varphi}=0$ for $\varphi \in \operatorname{Hom}_{\mathbb{Z}}\left(G, Z_{0}\right)$. Then $\left.\varphi\right|_{\operatorname{supp} A}=0$ follows and hence $\varphi=0$ because of our assumption $G=\langle\operatorname{supp} A\rangle$.

In the remainder of this section we will introduce division graded versions of the standard examples of Jordan algebras.

2.10. Proposition. Let $A$ be a division graded associative algebra over a field $F$ of ch. $F \neq 2$.

(a) Any graded subalgebra $J$ of $A^{+}$is a division $G$-graded Jordan algebra as soon as $x^{-1} \in J$ for any nonzero homogeneous $x \in J$. In particular, $A^{+}$itself is division G-graded.

(b) If $A$ is associatively generated by a division graded subalgebra $J$ of $A^{+}$, then

$$
Z(J)=Z(A) \cap J .
$$

(c) For $J=A^{+}$we have

$$
\operatorname{Der}_{F} A=\operatorname{Der}_{F} A^{+}, Z(A)=Z\left(A^{+}\right)
$$

and

$$
\operatorname{CDer}_{F} A=\operatorname{CDer}_{F} A^{+} \text {. }
$$

If $A$ is semiprime, then (b) follows from [22, Thm. 3.3]. However, $A$ is in general not semiprime. For example, even a group algebra over a field need not be semiprime (see [18 (10.17)]). Also, for an arbitrary prime algebra $A$, the equality $\operatorname{Der}_{F} A=$ $\operatorname{Der}_{F} A^{+}$follows from [13, Thm. 3.3]. Although $A$ is in general not prime, we can nevertheless use some of the methods of Herstein's proof. In particular, the crucial formula (2.10.4) below is taken from [13.

Proof. (a) follows from the fact that invertibility in $A$ and $A^{+}$are the same. For (b), the inclusion $Z(A) \cap J \subset Z(J)$ is obvious from (1.11.4). To prove the other inclusion, we may assume that $0 \neq z \in Z(J)$ is a homogeneous element. For any $x \in J$ we then have $[x, z]^{2}=2 x \cdot U_{z} x-U_{x} z^{2}-U_{z} x^{2}=0$ since $z \in Z(J)$. If $z \notin Z(A)$, then $[x, z] \neq 0$ for some homogeneous $x \in J$ and hence $[x, z]$ is invertible, contradicting $[x, z]^{2}=0$.

(c) It is clear that Der $A \subset \operatorname{Der} A^{+}$. So, let $d \in \operatorname{Der} A^{+}$and put $a^{b}=d(a b)-$ $d(a) b-a d(b)$ for $a, b \in A$. By calculating $d((a b) a b+b a(a b))$ in two different ways, one obtains [13. Lemma 3.6]

$$
a^{b}[a, b]=0 .
$$

Linearizing in $b$ gives

$$
a^{b}[a, c]+a^{c}[a, b]=0 .
$$

It suffices to prove $a^{b}=0$ for all homogeneous $a, b \in A$. We will do so by distinguishing four cases.

Case 1) $a, b \in Z(A)$ : Then $d(a b)=d(a . b)=d(a) . b+a . d(b)=d(a) b+a d(b)$, i.e., $a^{b}=0$. Case 2) $a \notin Z(A), b \in Z(A):$ By (2.10.5) we then have $a^{b}[a, c]=0$ for all $c \in A$. Since $a$ is homogeneous, there exists a homogeneous $c$ such that $[a, c] \neq 0$ and hence $[a, c]$ is invertible, forcing $a^{b}=0$. Case 3$) a \in Z(A), b \notin Z(A)$ : Since $a^{b}=-b^{a}$, this case follows from the previous one. Case 4) $a, b \notin Z(A)$ : If $[a, b] \neq 0$ we obtain $a^{b}=0$ from (2.10.4). Otherwise, we get $a^{b}[a, c]=0$ from (2.10.5) for all $c \in A$, and again $a^{b}=0$ follows. 
Thus $\operatorname{Der}_{F} A=\operatorname{Der}_{F} A^{+}$. That $Z(A)=Z\left(A^{+}\right)$follows from (2.10.1) and then $\operatorname{CDer}_{F} A=\operatorname{CDer}_{F} A^{+}$by definition.

2.11. Quadratic form Jordan algebras. Let $\Phi$ be an associative commutative ring, $M$ a $\Phi$-module and $q: M \rightarrow \Phi$ a quadratic form with base point, i.e., $q(1)=1$ for some $1 \in M$. We let $q(x, y)=q(x+y)-q(x)-q(y)$ be the polar of $q$, and define $\bar{x}=q(x, 1) 1-x$ and $U_{x} y=q(x, \bar{y}) x-q(x) \bar{y}$. Then $M$ together with the quadratic operator $U$ is a (quadratic) Jordan algebra denoted by $J$ over $\Phi$. We will often confuse $J$ with $M$. It is well known that any $x \in M$ with invertible $q(x)$ is invertible in $J$. Indeed, its inverse is $x^{-1}=\frac{1}{q(x)} \bar{x}$.

Suppose $\Phi$ is $G$-graded, $M$ is a graded module of type $G$ and $q: M \rightarrow \Phi$ is graded in the sense that $q\left(M_{g}\right) \subset \Phi_{2 g}, q\left(M_{g}, M_{h}\right) \subset \Phi_{g+h}$ for $g, h \in G$ and $1 \in M_{0}$. The corresponding Jordan algebra $J$ is then $G$-graded, i.e., $U_{J_{g}} J_{h} \subset J_{2 g+h}$ and $\left\{J_{g} J_{h} J_{i}\right\} \subset J_{g+h+i}$ where $\{\cdots\}$ is the Jordan triple product of $J$. Moreover, $J$ is division $G$-graded if $q$ is anisotropic graded in the sense that $q\left(m_{g}\right)$ is invertible for any $0 \neq m_{g} \in M_{g}=J_{g}$.

If $\frac{1}{2} \in \Phi$ we have $J=\Phi .1 \oplus V$ for $V=\{x \in M \mid q(x, 1)=0\}$ and with respect to this decomposition the bilinear product of $J$ satisfies

$$
\left(s_{1} 1 \oplus v_{1}\right)\left(s_{2} 1 \oplus v_{2}\right)=\left(s_{1} s_{2}+f\left(v_{1}, v_{2}\right)\right) 1 \oplus\left(s_{1} v_{2}+s_{2} v_{1}\right)
$$

for $f=-\left.\frac{1}{2} q(\cdot, \cdot)\right|_{V \times V}$. In this case, the associator of $x_{i}=s_{i} .1 \oplus v_{i}$ is, with obvious notation,

$$
\left(s_{1} \oplus v_{1}, s_{2} \oplus v_{2}, s_{3} \oplus v_{3}\right)=f\left(v_{1}, v_{2}\right) v_{3}-f\left(v_{3}, v_{2}\right) v_{1} \in V .
$$

Hence $\Phi .1 \subset Z(J)$ and $(J, J, J) \subset V$. We note that $J$ always has a nonzero invariant form, namely $(x \mid y)=q(x y, 1)$ is such a form. But in general this form is not graded. We now describe special classes of quadratic form algebras.

2.12. Lemma. Let $J$ be a quadratic form algebra over a G-graded $\Phi$ containing $\frac{1}{2}$, and decompose the underlying module $M=\Phi .1 \oplus V$ as in section 2.11 above. Suppose that $V$ is an orthogonal sum of nonzero $G$-graded $\Phi$-modules $V^{(i)}, i \in I$ with $|I| \geq 2$ such that each $q \mid V^{(i)}$ is anisotropic graded (hence the type $S_{i}$ of $V^{(i)}$ is contained in $G$ ). We extend the grading of $V$ to $M$ in the canonical way, so that $J$ is a $G$-graded algebra.

(a) Then $Z(J)=\Phi .1$ and $(J, J, J)=V$; hence $J=Z(J) \oplus(J, J, J)$.

(b) Let $\mathfrak{o}(f)=\left\{d \in \operatorname{End}_{\Phi} V \mid f(d u, v)+f(u, d v)=0\right.$ for all $\left.u, v \in V\right\}$ be the orthogonal Lie algebra associated to $f$ and let $\mathfrak{e o}(f)$ be the ideal spanned by all elementary orthogonal transformations $\mathbb{E}_{u, v}, u, v \in V$, given by $\mathbb{E}_{u, v}(w)=$ $f(u, w) v-f(v, w) u=(u, w, v)=\Delta_{v, u}(w)$. Then

$$
\operatorname{Der}_{\Phi} J \rightarrow \mathfrak{o}(f):\left.d \mapsto d\right|_{V}
$$

is an isomorphism of Lie algebras mapping IDer $J$ onto $\mathfrak{e}(f)$.

(c) Suppose $\Phi$ is division graded, so that by Lemma 2.8 every $V^{(i)}$ is free and has a homogeneous $\Phi$-basis. Assume that $V$ has finite rank with a homogeneous $\Phi$-basis $\left\{v_{1}, \ldots, v_{n}\right\}$ satisfying $f\left(v_{i}, v_{j}\right)=0$ for $i \neq j$. Then $\mathfrak{o}(f)=\mathfrak{e o}(f)$, and hence all $\Phi$-linear derivations are inner. Moreover, $\left\{\mathbb{E}_{v_{i}, v_{j}} \mid 1 \leq i<j \leq n\right\}$ is a $\Phi$-basis of $\mathfrak{o}(f)$.

Proof. At least in special cases, this result is known to the experts. For the convenience of the reader we include a short proof. 
(a) Let $z \in Z(J) \cap V$. By (2.11.2) we then have $0=(z, u, v)=f(z, u) v$ for any $u, v$ in different submodules $V^{(i)}$. Considering homogeneous components then forces $z=0$. Hence $Z(J)=\Phi$.1. If $u, v$ belong to the same $V^{(i)}$ but $w$ belongs to a different $V^{(j)}$, we get $(u, v, w)=f(u, v) w$. Since $f(u, v)$ for suitable choices of $u, v$ is invertible, this shows that $w \in(V, V, V)$, and then $V=(J, J, J)$ follows.

(b) Any $\Phi$-linear derivation $d$ annihilates $1 \in J$ and leaves $V=(J, J, J)$ invariant. That $\left.d\right|_{V} \in \mathfrak{o}(f)$ is immediate from the product formula (2.11.1). Thus the map (2.12.1) is well-defined and injective. That it is also surjective is an easy exercise. In case $\Phi$ is a field, (c) is well known. In the setting of this lemma, it is proven in [8].

2.13. Construction of a division graded quadratic form Jordan algebra. For easier reference we describe a special case of the situation considered in Lemma 2.12. This setting will be specialized in section 4.5 to define Clifford tori. Our construction uses the following data:

(i) $\Phi$ is a division graded commutative associative ring with grading group $H=\operatorname{supp} \Phi$ and $\frac{1}{2} \in \Phi_{0}$;

(ii) $H$ is a subgroup of an abelian group $G$ such that $G / H$ is a 2-group, i.e., any element of $G / H$ has order $\leq 2$;

(iii) $I \subset G$ is a set of representatives of some cosets of $G$ modulo $H$ different from $H$, with at least two elements;

(iv) $\left(z_{i}\right)_{i \in I}$ is a family of nonzero (hence invertible) elements $z_{i} \in \Phi_{2 i}$.

Assuming these data, we put $z_{0}=1 \in \Phi_{0}$ and let $V^{(i)}, i \in I \cup\{0\}$, be the graded $\Phi$-module of type $i+H$ and rank 1 with basis $v_{i} \in V_{i}^{(i)}=\Phi_{0} v_{i}$ where $v_{0}=z_{0}=1 \in \Phi_{0}$. Thus

$$
V^{(i)}=\bigoplus_{h \in H} V_{i+h}^{(i)} \text { with } V_{i+h}^{(i)}=\Phi_{h} v_{i}
$$

For $i \in I \cup\{0\}$ we let $q^{(i)}: V^{(i)} \rightarrow \Phi$ be the $\Phi$-quadratic form given by $q^{(i)}\left(v_{i}\right)=$ $z_{i} \in \Phi_{2 i}$. We then have a $G$-graded $\Phi$-module $M=\bigoplus_{i \in I \cup\{0\}} V^{(i)}=\bigoplus_{g \in G} M_{g}$, where

$$
M_{g}= \begin{cases}V_{i+h}^{(i)} & \text { if } g=i+h \text { for } i \in I \cup\{0\}, h \in H, \\ 0 & \text { otherwise }\end{cases}
$$

with an anisotropic graded quadratic form $q=\bigoplus_{i \in I \cup\{0\}} q^{(i)}$, the orthogonal sum of the quadratic forms $q^{(i)}$. Hence the corresponding quadratic form Jordan algebra $J$, considered as an algebra over $F=\Phi_{0}$, is division $G$-graded with supp $J=I+H$. Moreover, Lemma 2.12 applies. In particular, it follows from (1.13.2) and 2.12(a) that

$$
\operatorname{dim}_{F} \operatorname{GIF}(J)=1 .
$$

Since by construction $\Delta_{v_{i}, v_{j}} \in(\text { IDer } J)_{i+j}$ we obtain, using Lemma 2.8, the following more precise description of the inner derivation algebra.

2.14. Lemma. In the setting of section 2.13 suppose $I$ is finite, and let $<$ be a well-ordering on $I$. Also, denote by $\bar{g}$ the canonical image of $g$ in $G / H$.

Then $\left\{\Delta_{v_{i}, v_{j}} \mid i, j \in I, i<j\right\}$ is a $\Phi$-basis of $\mathrm{IDer} J=\operatorname{Der}_{\Phi} J$, and $\left\{\Delta_{v_{i}, v_{j}}: i, j \in\right.$ $I, i<j, \bar{i}+\bar{j}=\bar{g}\}$ is a $\Phi$-basis of $\bigoplus_{h \in H}(\operatorname{IDer} J)_{g+h}=(\operatorname{IDer} J)_{\bar{g}}$. In particular,

$$
\operatorname{dim}_{F}(\operatorname{IDer} J)_{g}=|\{\{i, j\}: i, j \in I, i \neq j, \bar{i}+\bar{j}=\bar{g}\}| .
$$


In a special case, these dimensions have been calculated in [27, Lemma 2.4].

2.15. Graded Albert algebras. Let $A$ be a prime associative algebra over $F$ whose central closure $\bar{A}$ has (generic) degree 3 , and let $\mu \in Z=Z(A)$ be invertible. Assume that $\operatorname{tr}(A) \subset Z$ where $\operatorname{tr}$ is the generic trace of $\bar{A}$. Then, the subset $J=(A, \mu):=A \oplus A \oplus A$ of the first Tits construction $(\bar{A}, \mu)=\bar{A} \oplus \bar{A} \oplus \bar{A}$ is a $Z$ subalgebra such that $(\overline{A, \mu})=(\bar{A}, \mu)([31$ Lemma 6.5]). Hence $J$ and $\bar{J}$ are Albert algebras in the sense of [21]. Since $\bar{J}=(\bar{A}, \mu)$ has degree 3 , Schafer's theorem [14] Exercise 1, p. 319] implies $\bar{J}=\bar{Z} \oplus(\bar{J}, \bar{J}, \bar{J})$ if ch. $F \neq 3$.

To obtain a grading on $J$ we assume the following data:

(i) $A=\bigoplus_{g \in G} A_{g}$ is a $G$-graded prime associative algebra with centre $Z$;

(ii) $|G / S|=3$ where $S=\operatorname{supp} A$;

(iii) $\mu \in Z_{3 g_{0}}$ for some $g_{0} \in G \backslash S$;

(iv) $\operatorname{tr}$ is $G$-graded, i.e., $\operatorname{tr}\left(A_{g}\right) \subset Z_{g}$ for all $g \in G$.

Then $J=(A, \mu)$ is $G$-graded with homogeneous spaces defined as follows:

$$
J_{g}:= \begin{cases}A_{g} \oplus 0 \oplus 0 & \text { if } g \in S, \\ 0 \oplus A_{g-g_{0}} \oplus 0 & \text { if } g-g_{0} \in S, \\ 0 \oplus 0 \oplus A_{g+g_{0}} & \text { if } g+g_{0} \in S .\end{cases}
$$

Moreover, $J$ is division graded if $A$ is so. If, in addition, $G$ is totally ordered and ch. $F \neq 3$, then $J=Z \oplus(J, J, J)$. Indeed, since $(\bar{J}, \bar{J}, \bar{J})=\bar{Z} \otimes_{Z}(J, J, J)$, this follows from Schafer's Theorem and 2.8(c) applied to the $Z$-modules $Z+(J, J, J)$ and $Z \cap(J, J, J)$.

\section{3. $G$-TORI}

In this section we will introduce a special class of division graded algebras, socalled $G$-tori. Our main interest will be the study of their derivation algebras. The basic assumptions of the previous sections remain in place: we consider unital alternative and Jordan algebras over a field $F$, which in the case of Jordan algebras will be assumed to have ch. $F \neq 2$.

3.1. $G$-tori. Let $G$ be an abelian group. A division $G$-graded algebra $T=\bigoplus_{g \in G} T_{g}$ over $F$ is called a $G$-torus if

(i) $\operatorname{dim}_{F} T_{g} \leq 1$ for all $g \in G$, and

(ii) $\operatorname{supp} T$ generates $G$ as an abelian group.

If the $G$-torus is associative, alternative or Jordan, it is called an associative, an alternative or a Jordan $G$-torus. We will frequently identify $T_{0}=F$. We say that a $G$-torus is of strong type if $T$ is strongly graded. In this case, the support $\operatorname{supp} T$ is a subgroup of $G$. By 2.3(a), any alternative $G$-torus is of strong type. An associative $G$-torus is the same as a twisted group algebra $F^{t}[G]$. In particular, if $T$ is a $G$-torus and $H$ its central grading group, the centre $Z(T)$ is a twisted group algebra of $H$. An example of a Jordan $G$-torus is the quadratic form Jordan algebra of section 2.13, viewed as an algebra over $F=\Phi_{0}$. 
3.2. Theorem. Let $T$ be a $G$-torus with central grading group $H$. Recall that $\mathcal{D}$ denotes the degree derivations of $T$; see (1.8.5). Then:

$$
\begin{aligned}
\left(\operatorname{Der}_{F} T\right)_{0} & =\mathcal{D}, \\
\left(\operatorname{CDer}_{F} T\right)_{\text {fin }} & =\bigoplus_{h \in H}\left(\operatorname{Der}_{F} T\right)_{h}=\left(\operatorname{grDer}_{F} T\right)_{H}, \\
\operatorname{IDer} T & \subset \bigoplus_{g \notin H}\left(\operatorname{Der}_{F} T\right)_{g}, \\
\left(\operatorname{CDer}_{F} T\right)_{\text {fin }} \cap \operatorname{IDer} T & =0 .
\end{aligned}
$$

If $G$ is finitely generated, then so is $T$, and hence $\operatorname{Der}_{F} T=\operatorname{grDer}_{F} T$. In this case we also have $\operatorname{CDer}_{F} T=\left(\operatorname{CDer}_{F} T\right)_{\text {fin }}$, which is a generalized Witt algebra by Proposition [2.9.

Proof. Let $d \in\left(\operatorname{Der}_{F} T\right)_{0}$ and define scalars $\varphi_{g}$ for $g \in S=\operatorname{supp} T$ by $d\left(t_{g}\right)=\varphi_{g} t_{g}$, $t_{g} \in T_{g}$. The fact that $d$ is a derivation means

$$
\varphi_{g}+\varphi_{h}=\varphi_{g+h} \quad \text { for } g, h \in S \text { with } T_{g} T_{h} \neq 0 .
$$

In particular, if $T$ is alternative, it is a torus of strong type with $S=G$ and we have $\varphi \in \operatorname{Hom}_{\mathbb{Z}}\left(G, Z_{0}\right)$, which means that $d$ is a degree derivation. For a Jordan torus $T$ we have $d U_{x}=U_{d x, x}+U_{x} d$ for $x \in T$, which implies

$$
\varphi_{2 g+h}=2 \varphi_{g}+\varphi_{h} \quad \text { for } g, h \in S .
$$

Since $\varphi_{0}=0$, it follows from (3.2.5) that $\varphi_{-h}=-\varphi_{h}$ and then 3.2.6 shows that $\varphi: S \rightarrow F$ is a homomorphism of reflection spaces preserving the base points. By Proposition 2.2(b), $\varphi$ extends uniquely to a group homomorphism $\Phi: G \rightarrow F$ and so $d$ is a degree derivation also in this case. This implies (3.2.1) and then (3.2.2) is immediate from (2.9.2) and the definition of $\left(\operatorname{grDer}_{F} T\right)_{H}$.

Since IDer $T$ is $G$-graded, it suffices to show that IDer $T \cap\left(\operatorname{Der}_{F} T\right)_{h}=0$ for all $h \in H$. Suppose there exists a nonzero $d \in\left(\operatorname{Der}_{F} T\right)_{h}$ for some $h \in H$. If $T$ is Jordan, we can assume that $d=\left[L_{x}, L_{y}\right]$, where $x \in T_{g}, y \in T_{g^{\prime}}$ and $g+g^{\prime}=h$. Hence $y=z x^{-1}$ for some nonzero $z \in Z_{h}$. But then $\left[L_{x}, L_{y}\right]=\left[L_{x}, L_{z x^{-1}}\right]=$ $L_{z}\left[L_{x}, L_{x}^{-1}\right]=0$, a contradiction. The proof for alternative algebras is similar, using the form of inner derivations (see section 1.4). Thus (3.2.3) holds, and this implies (3.2.4). The last statement follows from Lemma2.4, (1.6.2), and (1.7.4).

Remark. We have

$$
\operatorname{grDer}_{F} T=\operatorname{IDer} T \rtimes\left(\operatorname{CDer}_{F} T\right)_{\text {fin }}
$$

as soon as any $Z$-linear derivation is inner.

Indeed, by the result above, we only have to show $\left(\operatorname{Der}_{F} T\right)_{g} \subset$ IDer $T$ for $g \notin H$. But any $d \in\left(\operatorname{Der}_{F} T\right)_{g}$ has $d(Z) \subset Z \cap \bigoplus_{g^{\prime} \in g+H}\left(\operatorname{Der}_{F} T\right)_{g^{\prime}}=0$, and so is $Z$-linear.

By Lemma 2.12 the criterion (3.2.7) is fulfilled for the $G$-torus of section 2.13 with a finite $I$. This result is generalized in $[8$. Other examples of $G$-tori satisfying (3.2.7) will be discussed in Proposition 3.5. In the next section, we will use this criterion to establish $\operatorname{Der}_{F} T=\operatorname{IDer} T \rtimes \operatorname{CDer}_{F} T$ for Jordan $\mathbb{Z}^{n}$-tori, even if IDer $T=\operatorname{Der}_{Z} T$ is not always true there. 
3.3. Proposition. Let $T$ be an alternative $G$-torus with central grading group $H$. Then

$$
(T, T, T) \subset[T, T]=\bigoplus_{g \notin H} T_{g}
$$

hence

$$
T=Z(T) \oplus[T, T] \quad \text { and } \quad \mathcal{G} \mathcal{I} \mathcal{F}(T) \cong T_{0}^{*}
$$

is 1-dimensional. If $T$ is associative, we have

$$
\operatorname{IDer}_{F} T=\operatorname{IDer}_{F} T^{+} \cong \bigoplus_{g \notin H} T_{g} .
$$

Proof. For easier notation we put $B=\bigoplus_{g \notin H} T_{g}$. We will first prove that $(T, T, T) \subset$ $B$. Observe that $(T, T, T)$ is $G$-graded and invariant under $Z=Z(T)$. Hence, either $(T, T, T) \subset B$ or there exist homogeneous $a, b, c \in T$ such that $1=(a, b, c)$. Then $(a b)^{-1}$ and $c$ have the same degree, and therefore $c=z(a b)^{-1}=z b^{-1} a^{-1}$ for some nonzero $z \in T_{0}=Z_{0}$ by Lemma $2.8(\mathrm{a})$. But then we obtain the contradiction $1=z(a b)(a b)^{-1}-z a\left(b\left(b^{-1} a^{-1}\right)\right)=z-z=0$. Similarly, if $[T, T]$ is not contained in $B$, we have $1=[a, b]$ for suitable homogeneous $a, b \in T$, where $b=z^{\prime} a^{-1}$ for some $z^{\prime} \in Z_{0}$. Then $1=\left[a, a^{-1}\right] z^{\prime}=0$ gives a contradiction.

For (3.3.1) it remains to prove that $B \subset[T, T]$. By Lemma 2.5, any nonzero $a \in$ $B$ satisfies $[a, T] \neq 0$. It is of course not harmful to assume that $a$ is homogeneous. Then there exists a homogeneous $b \in T$ such that $0 \neq a b-b a=a b-(b a)\left(b^{-1} b\right)=$ $a b-\left(b\left(a b^{-1}\right)\right) b$ (by the Moufang identity) $=\left(a-b\left(a b^{-1}\right)\right) b$. Since $b$ is invertible, it follows that $0 \neq a-b\left(a b^{-1}\right)=z a$ for some $0 \neq z \in Z_{0}$. But then $\left[a b^{-1}, z^{-1} b\right]=$ $z^{-1}\left(\left(a b^{-1}\right) b-b\left(a b^{-1}\right)\right)=z^{-1}(z a)=a \in[T, T]$ proving our result.

The first equation in (3.3.2) is obvious, and the second follows from (1.13.2). For an associative $T$ we always have IDer $T=\operatorname{ad} T \cong T / Z(T)$ and $\operatorname{IDer} T^{+}=$ $\operatorname{ad}[T, T] \cong T /(Z(T) \cap[T, T])$.

3.4. Corollary. Let $A$ be an associative $G$-torus over a field $F$ of ch. $F \neq 2$ and let * be a graded F-linear involution of $A$ such that $J:=H(A, *)$ is a generating set of the associative algebra $A$. Denote by $H$ the central grading group of $A$. Then there is a graded isomorphism of Lie algebras

$$
\text { IDer } J \cong[J, J]=\bigoplus_{g \in G \backslash(H \cup \operatorname{supp} J)} A_{g}(\text { graded isomorphism })
$$

and hence for all $g \in G$,

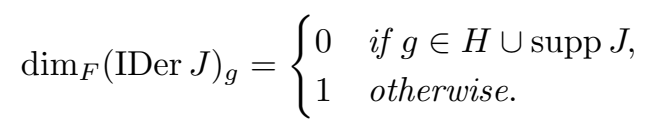

Proof. Because of (1.4.3) we have an epimorphism

$$
[J, J] \rightarrow \operatorname{IDer} J:\left.x \mapsto \operatorname{ad} x\right|_{J}
$$

of graded Lie algebras. Its kernel consists of all $x \in[J, J]$ that commute with $J$ and hence also with $A$. But then $x \in Z(A)$ so that (3.3.2) shows that $x=0$. Hence (3.4.3) is an isomorphism. For the proof of the second part of (3.4.1) let $S(A, *)=\left\{a \in A \mid a^{*}=-a\right\}$. Then $A=J \oplus S(A, *)$ and $[J, J] \subset S(A, *)=$ $\bigoplus_{g \notin \text { supp } J} A_{g}$ follows. By (3.3.2) we also have $[J, J] \subset \bigoplus_{g \notin H} A_{g}$. Conversely, for $g \notin(H \cup \operatorname{supp} J)$ and $0 \neq y \in A_{g}$ we get $[y, x] \neq 0$ for some $x \in J_{j}=A_{j}$ because 
$[y, J]=0$ would imply $y \in Z(A)=\bigoplus_{h \in H} A_{h}$. Observe that $[y, x] \in J$ and hence $g+j \in \operatorname{supp} J$. Because $\operatorname{supp} J$ is a reflection subspace of $G$, we then obtain $g-j=0 \cdot(j \cdot(g+j)) \in \operatorname{supp} J$ (of course, this can also be seen directly). But $[y, x] \neq 0$ implies $\left[y x^{-1}, x\right] \neq 0$. So $y x^{-1} \in J_{g-j}$ and $0 \neq\left[y x^{-1}, x\right] \in A_{g} \subset[J, J]$ follows. Finally, (3.4.2) follows immediately from (3.4.1).

3.5. Proposition. Let $T$ be an associative $G$-torus where $G$ is finitely generated and let $H$ be the central grading group.

(a) (Osborn-Passman 24, Cor. 2.3]) The derivation algebra of $T$ is a semidirect product

$$
\operatorname{Der}_{F} T=\operatorname{IDer} T \rtimes \operatorname{CDer} T,
$$

where

$$
\operatorname{CDer}_{F} T=\left(\operatorname{CDer}_{F} T\right)_{\text {fin }}=\left(\operatorname{grDer}_{F} T\right)_{H}=\bigoplus_{h \in H}\left(\operatorname{Der}_{F} T\right)_{h}
$$

and

$$
\operatorname{IDer} T=\bigoplus_{g \notin H}\left(\operatorname{Der}_{F} T\right)_{g} \cong \bigoplus_{g \notin H} T_{g} .
$$

(b) For the associated Jordan algebra $T^{+}$we have $Z(T)=Z\left(T^{+}\right)$,

$\operatorname{CDer}_{F} T^{+}=\operatorname{CDer}_{F} T$ and $\operatorname{IDer} T^{+}=\operatorname{IDer} T$,

and hence also $\operatorname{Der}_{F} T^{+}=\operatorname{IDer} T^{+} \rtimes \operatorname{CDer} T^{+}$is a semidirect product.

Proof. (a) Equation (3.5.1) is proven in [24, loc. cit.]. The remaining equations follow from (1.7.4), (3.2.2) and (3.2.3).

(b) is a corollary to (a), (2.10.2) and (3.3.3)

3.6. Proposition. Let $T$ be an associative $G$-torus over a field $E$ with ch. $E \neq 2$ and let $H$ be the central grading group of $T$. Let $J=\bigoplus_{g \in G} J_{g} \subset T^{+}$be a Jordan $G$ torus over a subfield $F$ of $E$, and assume that $J$ is a generating set of the associative algebra $T$. Then $Z(J)=Z(T) \cap J=\bigoplus_{h \in H} J_{h}$ and $(J, J, J)=\bigoplus_{h \notin H} J_{h}$. In particular,

$$
J=Z(J) \oplus(J, J, J) \quad \text { and } \quad \mathcal{G} \mathcal{I} \mathcal{F}(J) \cong J_{0} .
$$

Proof. By Proposition 3.3 we have $\bigoplus_{g \notin H} T_{g}=[T, T]=[T, Z(T)+[T, T]]=$ $[T,[T, T]]$. Because of (1.11.4) this implies $(J, J, J) \subset J \cap[T, T]=\bigoplus_{g \notin H} J_{g}$. For the other inclusion, let $0 \neq x \in J_{g}, g \notin H$. Then $x \notin Z(T)$ and there exists a homogeneous $y \in J$ such that $[x, y] \neq 0$. Hence, $y x=z x y$ for some $0 \neq z \in E$. Then $\left(x, y, y^{-1}\right)=\frac{1}{4}\left(2+z+z^{-1}\right) x \in J_{g}$, i.e., $\left(x, y, y^{-1}\right)=t x$ for some nonzero $t \in F$, whence $x \in(J, J, J)$.

3.7. Corollary. Let $A$ be an associative $G$-torus over a field $E$ of ch. $E \neq 2$. Suppose that $G$ is finitely generated and that $*$ is a graded involution of the second kind. With the notation of Example 1.11, we then have for the Jordan G-torus $H=H(A, *)$ over $F$ :

(a) $H=Z(H) \oplus(H, H, H)$;

(b) $\operatorname{Der}_{F} H=$ IDer $H \rtimes$ CDer $H$.

Proof. (a) is a special case of Proposition 3.6. For (b) we have $\operatorname{Der}_{E} A^{+}=\operatorname{IDer} A^{+} \rtimes$ CDer $A^{+}$by Proposition 3.5. and both summands are left invariant under the automorphism $d \mapsto d^{*}$ of $\operatorname{Der}_{E} A^{+}$. By (1.11.9) and (1.11.7) the fixed point spaces under $*$ are the corresponding subalgebras for $H$, whence the result. 


\section{4. $\mathbb{Z}^{n}$-TORI}

4.1. Tori. A $\mathbb{Z}^{n}$-torus will be called an $n$-torus or simply a torus. Hence an $n$ torus is a division $\mathbb{Z}^{n}$-graded algebra $T=\bigoplus_{\alpha \in \mathbb{Z}^{n}} T_{\alpha}$ such that $\operatorname{dim}_{F} T_{\alpha} \leq 1$ for all $\alpha \in \mathbb{Z}^{n}$ and $\operatorname{supp} T$ generates $\mathbb{Z}^{n}$. The reader is reminded that all algebras considered here are alternative or Jordan over a field $F$ of ch. $F \neq 2$ if $T$ is Jordan. In the following, the notion "torus" will always mean an alternative or a Jordan torus.

Let $T=\bigoplus_{\alpha \in \mathbb{Z}^{n}} T_{\alpha}$ and $T^{\prime}=\bigoplus_{\alpha \in \mathbb{Z}^{n}} T_{\alpha}^{\prime}$ be two $n$-tori. We will say that $T$ and $T^{\prime}$ are graded isomorphic, abbreviated as $T \cong_{\mathbb{Z}^{n}} T^{\prime}$, if there exists an isomorphism $\varphi: T \rightarrow T^{\prime}$ such that $\varphi\left(T_{\alpha}\right)=T_{\alpha}^{\prime}$ for all $\alpha \in \mathbb{Z}^{n}$.

We recall from section 2.3 that $\operatorname{supp} T=\mathbb{Z}^{n}$ if $T$ is alternative and that $\operatorname{supp} T$ is a semilattice in $\mathbb{Z}^{n}$ if $T$ is Jordan. Also, by Lemma 2.4.

$$
\text { any torus is finitely generated, }
$$

and any torus is a domain, and hence strongly prime $(2.3)(\mathrm{c}))$.

The structure of alternative tori has been determined up to graded isomorphism in [5. Thm. 1.25] and in improved form in [29 4.11]. Besides the associative tori, see section 4.2 below, there is one more type, the so-called Cayley torus in ch. $F \neq 2$; see (4.3.1).

4.2. Associative tori. An $n \times n$ matrix $q=\left(q_{i j}\right)$ over a field $F$ satisfying $q_{i i}=1$ and $q_{j i}=q_{i j}^{-1}$ is called a quantum matrix. For a quantum matrix $q$ the associated quantum torus $F_{q}=F_{q}\left[t_{1}^{ \pm 1}, \ldots, t_{n}^{ \pm 1}\right]$ is the associative algebra over $F$ with $2 n$ generators $t_{1}^{ \pm 1}, \ldots, t_{n}^{ \pm 1}$, and relations $t_{i} t_{i}^{-1}=t_{i}^{-1} t_{i}=1$ and $t_{j} t_{i}=q_{i j} t_{i} t_{j}$ for all $1 \leq i, j \leq n$. Note that $F_{q}$ is commutative if and only if $q=\mathbf{1}$ where $\mathbf{1}$ is the quantum matrix whose entries are all 1 . In this case, the quantum torus $F_{\mathbf{1}}$ becomes the algebra of Laurent polynomials $F\left[t_{1}^{ \pm 1}, \ldots, t_{n}^{ \pm 1}\right]$ in $n$ variables.

Let $\left\langle\sigma_{1}, \ldots, \sigma_{n}\right\rangle$ be a basis of $\mathbb{Z}^{n}$, and define the degree of $t_{\alpha}:=t_{1}^{\alpha_{1}} \cdots t_{n}^{\alpha_{n}}$, where $\alpha=\alpha_{1} \sigma_{1}+\cdots+\alpha_{n} \sigma_{n} \in \mathbb{Z}^{n}$, to be $\alpha$. Then $F_{q}=\bigoplus_{\alpha \in \mathbb{Z}^{n}} F t_{\alpha}$ is an $n$-torus. We call this grading a toral $\mathbb{Z}^{n}$-grading of $F_{q}$, or, if one needs to specify a basis of $\mathbb{Z}^{n}$, a $\left\langle\sigma_{1}, \ldots, \sigma_{n}\right\rangle$-grading of $F_{q}$.

It is shown in [5, 1.8] that any associative torus is graded isomorphic to some $F_{q}$ with a suitable toral grading. Any commutative associative torus is graded isomorphic to a Laurent polynomial ring $F_{\mathbf{1}}=F\left[t_{1}^{ \pm 1}, \ldots, t_{n}^{ \pm 1}\right]$.

4.3. Alternative tori. Suppose ch. $F \neq 2$. For $n \geq 3$ the Cayley $n$-torus

$$
\mathbb{O}_{t}=\left(F\left[t_{1}^{ \pm 1}, \ldots, t_{n}^{ \pm 1}\right], t_{1}, t_{2}, t_{3}\right)
$$

is the $F$-algebra obtained by the Cayley-Dickson process over the Laurent polynomial ring $F\left[t_{1}^{ \pm 1}, \ldots, t_{n}^{ \pm 1}\right]$, using the structure constants $t_{1}, t_{2}$ and $t_{3}$. This becomes an alternative torus with $\operatorname{supp} \mathbb{O}_{t}=\mathbb{Z}^{n}$ and centre $F\left[t_{1}^{ \pm 1}, \ldots, t_{n}^{ \pm 1}\right]$ graded by

$$
2 \mathbb{Z} \sigma_{1}+2 \mathbb{Z} \sigma_{2}+2 \mathbb{Z} \sigma_{3}+\mathbb{Z} \sigma_{4}+\cdots+\mathbb{Z} \sigma_{n}
$$

for a basis $\left\langle\sigma_{1}, \ldots, \sigma_{n}\right\rangle$ of $\mathbb{Z}^{n}$ with $\operatorname{deg} t_{i}=2 \sigma_{i}$ for $i=1,2,3$ and $\operatorname{deg} t_{i}=\sigma_{i}$ for $i=4, \ldots, n$.

We will next describe Jordan tori. For details, see [31]. 
4.4. Jordan tori of Hermitian type. Let $\varepsilon=\left(\varepsilon_{i j}\right)$ be an elementary quantum matrix, i.e., a quantum matrix with all $\varepsilon_{i j}=1$ or -1 . On the quantum torus $F_{\varepsilon}=F_{\varepsilon}\left[t_{1}^{ \pm 1}, \ldots, t_{n}^{ \pm 1}\right]$, there exists a unique involution $*$ such that $t_{i}^{*}=t_{i}$ for all $i$. The symmetric elements $H\left(F_{\varepsilon}, *\right)$ form a Jordan torus with respect to the grading induced from a toral grading of $F_{\varepsilon}$. One can show that $\operatorname{supp} H\left(F_{\varepsilon}, *\right)=\mathbb{Z}^{n}$ if and only if $q=\mathbf{1}$ if and only if $H\left(F_{\varepsilon}, *\right)$ is of strong type. If $q \neq \mathbf{1}$, the central grading group of $H\left(F_{\varepsilon}, *\right)$ is

$$
2 \mathbb{Z} \sigma_{1}+\cdots+2 \mathbb{Z} \sigma_{m}+\mathbb{Z} \sigma_{m+1}+\cdots+\mathbb{Z} \sigma_{n}
$$

for a suitable toral $\left\langle\sigma_{1}, \ldots, \sigma_{n}\right\rangle$-grading of $F_{\varepsilon}$ and $2 \leq m \leq n$ ([30, 2.5] and (2.10.1)), while by [30, 1.8] the central grading group of $F_{\varepsilon}$ is always of type

$$
2 \mathbb{Z} \sigma_{1}+\cdots+2 \mathbb{Z} \sigma_{2 l}+\mathbb{Z} \sigma_{2 l+1}+\cdots+\mathbb{Z} \sigma_{n} .
$$

Let $E$ be a quadratic field extension of $F$. Let $\sigma_{E}$ be the nontrivial Galois automorphism of $E$ over $F$ (recall that we assume ch. $F \neq 2$ for Jordan tori). Let $\xi=\left(\xi_{i j}\right)$ be a quantum matrix over $E$ such that $\sigma_{E}\left(\xi_{i j}\right) \xi_{i j}=1$, or, equivalently, $\sigma_{E}\left(\xi_{i j}\right)=\xi_{j i}$ for all $i, j$. On the quantum torus $E_{\xi}=E_{\xi}\left[t_{1}^{ \pm 1}, \ldots, t_{n}^{ \pm 1}\right]$ over $E$, there exists a unique $\sigma_{E}$-semilinear involution $\sigma$ such that $\sigma\left(t_{i}\right)=t_{i}$ for all $i$. The symmetric elements $H\left(E_{\xi}, \sigma\right)$ form a Jordan torus over $F$ with respect to the grading induced from a toral grading of $E_{\xi}$. We always have $\operatorname{supp} H\left(E_{\xi}, \sigma\right)=\mathbb{Z}^{n}$, and the central grading groups of $H\left(E_{\xi}, \sigma\right)$ and $E_{\xi}$ coincide.

4.5. Jordan tori of Clifford type. Let $2 \leq m \leq n$ and let $S^{(m)}$ be a semilattice in $\mathbb{Z}^{m}$. We consider the following data:

(i) $\Phi$ is the Laurent polynomial ring $\Phi=F\left[t_{1}^{ \pm 1}, \ldots, t_{n}^{ \pm 1}\right]$, which we view as a division $\mathbb{Z}^{n}$-graded algebra of $\operatorname{supp} \Phi=2 \mathbb{Z}^{m} \oplus \mathbb{Z}^{n-m}$ with respect to the canonical grading assigning $t_{i}$ the degree $(0, \ldots, 0,2,0, \ldots, 0)$ with 2 in the $i$ th component in case $1 \leq i \leq m$ and degree $(0, \ldots, 0,1,0, \ldots, 0)$ for $i>m$;

(ii) $I \subset \mathbb{Z}^{m} \subset \mathbb{Z}^{m} \oplus \mathbb{Z}^{n-m}=\mathbb{Z}^{n}$ is a set of representatives of $S^{(m)} / 2 \mathbb{Z}^{m}$, excluding the class $2 \mathbb{Z}^{m}$

(iii) $\left(z_{i}\right)_{i \in I}$ is a family of nonzero elements in $\Phi_{2 i}$.

The Clifford torus $J\left(S^{(m)},\left(z_{i}\right)_{i \in I}\right)$ is the quadratic form Jordan algebra constructed in section 2.13 using the data above. In particular, the results mentioned in Lemma 2.12, section 2.13, and Lemma 2.14 hold.

4.6. The Albert torus. Let $n \geq 3$. We assume that $F$ contains a primitive 3 rd root of unity $\omega$, in particular ch. $F \neq 3$, and denote by $w$ the quantum $(n \times n)$ matrix with $(1,2)$-entry equal to $\omega,(2,1)$-entry equal to $\omega^{-1}$ and all other entries equal to 1.

Let $F_{w}=F_{w}\left[u_{1}^{ \pm 1}, \ldots, u_{n}^{ \pm 1}\right]$ be the quantum torus determined by $w$, and let $Z=Z\left(F_{w}\right)$ be the centre of $F_{w}$. One finds $Z=F\left[u_{1}^{ \pm 3}, u_{2}^{ \pm 3}, u_{3}^{ \pm 1}, \ldots, u_{n}^{ \pm 1}\right]$, the algebra of Laurent polynomials in the variables $u_{1}^{3}, u_{2}^{3}, u_{3}, \ldots, u_{n}$. Let $\left\langle\sigma_{1}, \ldots, \sigma_{n}\right\rangle$ be a basis of $\mathbb{Z}^{n}$ and put

$$
S:=\mathbb{Z} \sigma_{1}+\mathbb{Z} \sigma_{2}+3 \mathbb{Z} \sigma_{3}+\mathbb{Z} \sigma_{4}+\cdots+\mathbb{Z} \sigma_{n} .
$$

We give $F_{w}$ a toral $S$-grading, i.e., $F_{w}=\bigoplus_{\sigma \in S} F u_{\sigma}$, where

$$
u_{\sigma}=u_{1}^{m_{1}} \cdots u_{n}^{m_{n}} \text { for } \sigma=m_{1} \sigma_{1}+m_{2} \sigma_{2}+3 m_{3} \sigma_{3}+m_{4} \sigma_{4}+\cdots+m_{n} \sigma_{n} .
$$


One knows ([31]) that $A=F_{w}$ fulfills the assumptions of section 2.15 for $G=\mathbb{Z}^{n}$ and $S$ as above. The exceptional Jordan algebra $\mathbb{A}_{t}=\left(F_{w}, u_{3}\right)$ of section 2.15$]$ is called the Albert torus. The grading of $\mathbb{A}_{t}$ defined in section[2.15] is called the toral grading. We note that

$$
\mathbb{A}_{t} \text { is of strong type. }
$$

4.7. Classification of Jordan tori [31]. Let ch.F $\neq 2$. Then any Jordan torus is graded isomorphic to one of the five tori

$$
F_{q}^{+}, H\left(F_{\varepsilon}, *\right), H\left(E_{\xi}, \sigma\right), J\left(S^{(m)},\left(z_{i}\right)_{i \in I}\right) \text { or } \mathbb{A}_{t}
$$

endowed with suitable toral gradings.

4.8. Central closures of tori. By section 2.3(d), the centre $Z$ of a torus $T$ is an integral domain, so that we can form the central closure $\bar{T}$ of $T$ (see section 1.2). It follows that $\bar{T}$ is always a central domain. By [31, 3.9], $\bar{T}$ is a $\mathbb{Z}^{n} / H$-torus over $\bar{Z}$, where $H$ denotes the central grading group. Moreover, we have

$$
\bar{T} \text { is a division algebra } \Leftrightarrow \bar{T} \text { is finite-dimensional. }
$$

Since both Jordan and alternative algebras are power associative, the implication $\Leftarrow$ is a special case of the general fact that a power associative finite-dimensional domain is a division algebra. To prove this general result, we consider the subalgebra generated by a single element and are then reduced to showing that a finite-dimensional associative domain is a division algebra. This is of course well known. It is, for example, an immediate consequence of Wedderburn's Structure Theorem. For the other direction, suppose that $\bar{T}$ is infinite-dimensional. Then the rank of the central grading group $H$ of $T$ is less than $n$. Hence the $\mathbb{Z}^{n} / H$-torus $\bar{T}$ contains a subalgebra which is a 1-torus. But a 1-torus is not a division algebra by [31, 3.6] for Jordan tori and [29, §2] in the alternative case, and then $\bar{T}$ is not a division algebra either.

We list here the central closures of Jordan tori and of the Cayley torus:

(a) The central closure of $F_{q}^{+}$is the +-algebra of $\overline{F_{q}}$, i.e., $\overline{F_{q}^{+}}=\bar{F}_{q}^{+}$. (This holds for any prime associative $A$ by 22 , Cor. 3.4]).

(b) By Lemma 1.3 the central closures of the Hermitian tori $H\left(F_{\varepsilon}, *\right)$ and $H\left(E_{\xi}, \sigma\right)$ are $H\left(\overline{F_{\varepsilon}}, *\right)$ and $H\left(\overline{E_{\xi}}, \sigma\right)$ respectively. Here $H\left(\overline{F_{\varepsilon}}, *\right)$ is a finite-dimensional central Jordan division algebra of degree $2^{m}$ for a suitable $m$. Indeed, by (4.4.2) and 4.8.1), $\overline{F_{\varepsilon}}$ is a central-simple division algebra of dimension $2^{2 l}$, hence of degree $2^{l}$. By the discussion in [14, p. 209] the degree of $H\left(\overline{F_{\varepsilon}}, *\right)$ is therefore either $2^{l}$ or $2^{l-1}$.

(c) The central closure of a Clifford torus $J\left(S^{(m)},\left(z_{i}\right)_{i \in I}\right)$ is an $(|I|+1)$-dimensional Jordan division algebra of a symmetric bilinear form.

(d) The central closure of an Albert torus is a 27-dimensional Albert division algebra over a field of characteristic $\neq 2,3$. Similarly, the central closure of the Cayley torus is an 8-dimensional octonion algebra over a field of characteristic $\neq 2$, hence a division algebra by (4.8.1).

As a consequence of the above, we have

$$
\operatorname{dim}_{\bar{Z}} \bar{T}<\infty \text { for } T=H\left(F_{\varepsilon}, *\right), J\left(S^{(m)},\left(z_{i}\right)_{i \in I}\right), \mathbb{O}_{t} \text { and } \mathbb{A}_{t} .
$$

We note that the central closures of $F_{q}, F_{q}^{+}$and $H\left(E_{\xi}, \sigma\right)$ are, in general, not finitedimensional over $\bar{Z}$. 
4.9. Proposition. For a Jordan torus $J$ over $F$ with ch. $F \neq 3$ in case $J$ is an Albert torus, we have $J=Z(J) \oplus(J, J, J)$. Hence $\mathcal{G} \mathcal{I} \mathcal{F}(J) \cong F 1$ is 1-dimensional.

Proof. This follows from (1.13.2) and the classification of Jordan tori in section 4.7. using Proposition 3.6 for $J=F_{q}^{+}, H\left(F_{\varepsilon}, *\right)$, Corollary 3.7 for $J=H\left(E_{\xi}, \sigma\right)$, (2.13.1) for $J=J\left(S^{(m)},\left(z_{i}\right)_{i \in I}\right)$ and section 2.15 for $J=\mathbb{A}_{t}$.

The analogous result for alternative $G$-tori is proven without classification in Proposition 3.3 .

4.10. Lemma. Let $T$ be a Jordan torus but not of type $F_{q}^{+}$or $H\left(E_{\xi}, \sigma\right)$ or let $T$ be a Cayley torus over a field $F$ of ch. $F \neq 2,3$. Then $\operatorname{IDer} T=\operatorname{Der}_{Z} T$.

Proof. For a Clifford torus this is a special case of Lemma2.12(c). For the remaining cases we use (11.6.1) and Lemma 2.6. So it suffices to show that $\bar{T}$ has only inner derivations. Given the structure of $\bar{T}$ described above, this is known. Indeed, for $T=H\left(F_{\varepsilon}, *\right)$ we can use [14 Exercise 1, p. 258] for algebras of degree 2 and [14. Theorem 9, p. 254] for algebras of degree $\geq 3$, for the Albert torus this follows from 14. Theorem 17, p. 408] and for the Cayley torus this is a consequence of [14 p. 301, Lemma 3] (see also [5, Lemma 1.39(b)]).

If $F_{q}, T=F_{q}^{+}$or $H\left(E_{\xi}, \sigma\right)$, then IDer $T=\operatorname{Der}_{Z} T$ is not true in general. For example, if $T$ is simple, then $\operatorname{Der}_{Z} T=\operatorname{Der}_{F} T$ because $Z=F$ in this case.

We are now ready to prove our main result.

4.11. Theorem. Let $T$ be an $n$-torus over a field $F$ and assume that ch. $F \neq 2$ if $T$ is a Jordan torus and that ch. $F \neq 2,3$ if $T$ is a Cayley torus or an Albert torus. Then

$$
\operatorname{Der} T=\operatorname{IDer} T \rtimes \operatorname{CDer} T \text {. }
$$

With respect to any nondegenerate graded invariant form we have

$$
\text { SDer } T=\operatorname{IDer} T \rtimes(\operatorname{SDer} T \cap \operatorname{CDer} T) .
$$

If $H \subset \mathbb{Z}^{n}$ is the central grading group of $T$, then

$$
\operatorname{IDer} T=\bigoplus_{\alpha \in \mathbb{Z}^{n} \backslash H}\left(\operatorname{Der}_{F} T\right)_{\alpha} \quad \text { and } \quad \operatorname{CDer} T=\bigoplus_{\alpha \in H}\left(\operatorname{Der}_{F} T\right)_{\alpha} .
$$

Proof. For $T \cong_{\mathbb{Z}^{n}} F_{q}$ and $T \cong_{\mathbb{Z}^{n}} F_{q}^{+}$, 4.11.1 has been proven in Proposition 3.5 and for $T \cong_{\mathbb{Z}^{n}} H\left(E_{\xi}, \sigma\right)$ in Corollary [3.7. The remaining cases follow from (3.2.7) and Lemma4.10 keeping in mind that $\operatorname{grDer}_{F} T=\operatorname{Der}_{F} T$ since $T$ is finitely generated. The structure of SDer $T$ is immediate from section 1.14, and (4.11.3) follows from Theorem 3.2

Remarks. For an alternative torus, (4.11.1) was known before. Indeed, an alternative torus is either a quantum torus, in which case this was proven in [4, Lemma 2.48 , p. 364] for the field $F=\mathbb{C}$ of complex numbers, but the proof works over any field or a Cayley torus, in which case (4.11.1) was proven for ch. $F \neq 2,3$ in [5, Theorem 1.40]. We have reproven the result here to show how it fits in the more general framework of tori considered in this paper, and also because it can be done without extra effort. Since (4.11.1) holds for Jordan and alternative tori, it is natural to conjecture that it also holds for structurable tori. 
The ideal IDer $T$ will be described in more detail in section 4.13, Lemma 4.14 and Theorem 4.15 below. The structure of the central derivation algebra CDer $T$ is determined in Theorem 4.12, Concerning SDer $T \cap \operatorname{CDer} T$, see section 1.14

4.12. Theorem. Let $T$ be an $n$-torus with centre $Z$ and central grading group $H$ of rank $r$. Then the central derivations CDer $T$ are isomorphic to the generalized Witt algebra associated to $Z=F[H], \mathcal{D} \cong \operatorname{Hom}_{\mathbb{Z}}\left(\mathbb{Z}^{n}, F\right)$ and the canonical pairing $p: \operatorname{Hom}_{\mathbb{Z}}\left(\mathbb{Z}^{n}, F\right) \times H \rightarrow F:(\Phi, h) \mapsto \Phi(h)$. Moreover,

$$
\text { CDer } T \cong \operatorname{Der}_{F} Z \ltimes Y,
$$

where

(i) $Z \otimes_{F} \operatorname{Hom}_{\mathbb{Z}}(H, F) \cong \operatorname{Der}_{F} Z \cong \operatorname{Der} F\left[z_{1}^{ \pm 1}, \ldots, z_{r}^{ \pm 1}\right]$ is a generalized Witt algebra and

(ii) $Y \cong Z \otimes_{F} \operatorname{Hom}_{\mathbb{Z}}\left(\mathbb{Z}^{n} / H, F\right)$ is an abelian ideal.

If $r \geq 1$ and ch. $F \neq 2$, then CDer $T$ is a perfect Lie algebra.

Proof. Since CDer $T_{\text {fin }}=$ CDer $T$ by Theorem 3.2, it follows from Proposition 2.9 that CDer $T$ is a generalized Witt algebra. Note that in our case the centre $Z$ is actually isomorphic to the group algebra of $H$ with a suitable grading. The splitting 4.12.1) will be a consequence of

$$
\operatorname{Hom}_{\mathbb{Z}}\left(\mathbb{Z}^{n}, F\right)=\operatorname{Hom}_{\mathbb{Z}}(H, F) \oplus \operatorname{Hom}_{\mathbb{Z}}\left(\mathbb{Z}^{n} / H, F\right) .
$$

To establish (4.12.2), we consider the canonical sequence of $F$-linear maps

$$
0 \rightarrow \operatorname{Hom}_{\mathbb{Z}}\left(\mathbb{Z}^{n} / H, F\right) \stackrel{\iota}{\rightarrow} \operatorname{Hom}_{\mathbb{Z}}\left(\mathbb{Z}^{n}, F\right) \stackrel{\varrho}{\rightarrow} \operatorname{Hom}_{\mathbb{Z}}(H, F) \rightarrow 0 .
$$

The imbedding $\iota$ is given by $\varphi \mapsto \varphi \circ \pi$, where $\pi: \mathbb{Z}^{n} \rightarrow \mathbb{Z}^{n} / H$ is the canonical epimorphism. The map $\varrho$ is the restriction map. Obviously, the image of $\iota$ equals the kernel of $\varrho$. We write $H=m_{1} \mathbb{Z} \varepsilon_{1}+\cdots+m_{n} \mathbb{Z} \varepsilon_{n}$, where $\left\langle\varepsilon_{1}, \ldots, \varepsilon_{n}\right\rangle$ is a $\mathbb{Z}$-basis of $\mathbb{Z}^{n}$ and where $m_{1}, \ldots, m_{n} \geq 0$. Then surjectivity of $\varrho$ will follow from

ch. $F$ does not divide $m_{i}$ for any nonzero $m_{i}$.

Indeed, for a Cayley torus or $H\left(F_{\varepsilon}, *\right)$ or a Clifford torus, all $m_{i}=1$ or 2 , and ch. $F \neq 2$ in these cases (see (4.3.1), (4.4.1), Lemma 2.12 and section 4.5). For an Albert torus, all $m_{i}=1$ or 3 , but also ch. $F \neq 3$ in this case (see section 4.6). Thus we are left with $F_{q}, F_{q}^{+}$and $H\left(E_{\xi}, \sigma\right)$. Since the central grading groups of $F_{q}$ and $F_{q}^{+}$, and the central grading groups of $E_{\xi}$ and $H\left(E_{\xi}, \sigma\right)$ coincide, we only need to consider the case $F_{q}=F_{q}\left[t_{1}^{ \pm 1}, \ldots, t_{n}^{ \pm 1}\right]$. In this case, the result is proven in [12. Lemma 3.6]. We include a short proof for the convenience of the reader. Suppose that $p:=\operatorname{ch} . F$ divides $m_{k} \neq 0$ for some $k$ and let $q=\left(q_{i j}\right)$. Then $t_{k}^{m_{k}} \in Z$, and so $t_{k}^{m_{k}} t_{j}=t_{j} t_{k}^{m_{k}}=q_{k j}^{m_{k}} t_{k}^{m_{k}} t_{j}$, whence $q_{k_{j}}^{m_{k}}=1$ for all $j$. Since $m_{k}=p l_{k}$ for some $l_{k} \geq 1$ and ch. $F=p$, we have $q_{k j}^{l_{k}}=1$ for all $j$. Hence $t_{k}^{l_{k}}$ commutes with all $t_{j}$, and we get $t_{k}^{l_{k}} \in Z$, but $l_{k} \varepsilon_{k} \notin H$, a contradiction.

Thus (4.12.4) holds and $\varrho$ is surjective. Hence (4.12.3) is exact and so (4.12.2) holds. By Lemma 1.10 we then have a decomposition

$$
\operatorname{CDer} T=\left(Z \otimes_{F} \operatorname{Hom}_{\mathbb{Z}}(H, F)\right) \ltimes\left(Z \otimes_{F} \operatorname{Hom}_{\mathbb{Z}}\left(\mathbb{Z}^{n} / H, F\right)\right)
$$

keeping in mind that $\mathcal{D} \cong \operatorname{Hom}_{\mathbb{Z}}\left(\mathbb{Z}^{n}, F\right)$ and hence $\mathcal{D}^{H} \cong \operatorname{Hom}_{\mathbb{Z}}\left(\mathbb{Z}^{n} / H, F\right)$. The structure of $Z \otimes_{F} \operatorname{Hom}_{\mathbb{Z}}(H, F)$ follows from Propositions [3.5(a) and [2.9. 
Since $\operatorname{Hom}_{\mathbb{Z}}\left(\mathbb{Z}^{n}, F\right) \cong F^{n}$, the right kernel of $p$ is trivial, and hence $p \neq 0$ as soon as $r \geq 1$. In particular, by 1.10(b), CDer $T$ is a perfect Lie algebra in this case.

We now turn to the description of IDer $T$. As one can see from [4] and [5], the knowledge of IDer $T$ will be useful in the classification of the extended affine Lie algebras with coordinate algebras $T$. For the convenience of the reader we first give a summary of the results on IDer $T$ obtained so far.

4.13. Summary of results on IDer $T$. Throughout, we let $T$ be an $n$-torus over a field $F$ satisfying the assumptions of Theorem 4.11 We denote by $Z$ the centre of $T$ and by $H$ the central grading group. We have seen in (4.11.3) that

$$
\operatorname{IDer} T=\bigoplus_{\alpha \in \mathbb{Z}^{n} \backslash H}\left(\operatorname{IDer}_{F} T\right)_{\alpha} .
$$

Concerning the Lie algebra structure, we note that by 1.4 .2 the Lie algebra IDer $T$ is a $Z$-form of IDer $\bar{T}$, where $\bar{T}$ is the central closure of $T$.

(a) Specializing Proposition 3.5 Corollary 3.7 and (1.11.7) we know that

$$
\operatorname{dim}_{F}(\operatorname{IDer} T)_{\alpha}=1 \text { for all } \alpha \in \mathbb{Z}^{n} \backslash H \text { and } T=F_{q}, F_{q}^{+} \text {or } H\left(E_{\xi}, \sigma\right) .
$$

Note that for $T=F_{q}^{+}$and $T=H\left(E_{\xi}, \sigma\right)$, the central grading group $H$ of $T$ coincides with the central grading group of $F_{q}$ and $E_{\xi}$, respectively. Of course, (4.13.2) for $T=F_{q}$ is already contained in [4, 2.55].

(b) By Corollary 3.4 we have a similar result for $T=H\left(F_{\varepsilon}, *\right)$. Denoting by $H_{\varepsilon}$ the central grading group of the quantum torus $F_{\varepsilon}$ we have

$$
\operatorname{dim}_{F}\left(\operatorname{IDer} H\left(F_{\varepsilon}, *\right)\right)_{\alpha}= \begin{cases}1 & \alpha \in \mathbb{Z}^{n} \backslash\left(H_{\varepsilon} \cup \operatorname{supp} H\left(F_{\varepsilon}, *\right)\right), \\ 0 & \text { otherwise. }\end{cases}
$$

We note that $H=H_{\varepsilon} \cap \operatorname{supp} H\left(F_{\varepsilon}, *\right)$ by (2.10.1) but $H_{\varepsilon} \not \subset \operatorname{supp} H\left(F_{\varepsilon}, *\right)$ in general.

(c) For the Cayley torus $\mathbb{O}_{t}$ where ch. $F \neq 3$, one knows from 5 . Theorem 1.40, p. 4328] that

$$
\operatorname{dim}_{F}\left(\operatorname{IDer} \mathbb{O}_{t}\right)_{\alpha}=2 \text { for all } \alpha \in \mathbb{Z}^{n} \backslash H .
$$

(d) The dimensions of (IDer $T)_{\alpha}$ in the case of a Clifford torus follow from the general formula (2.14.1). In particular, it is interesting to note that contrary to all other cases, the dimension varies with $\alpha$ and it can also get arbitrarily large. For example, in the setting of section 4.5, assume that $I=\left\{\sigma_{1}, \sigma_{2}\right\}$. Then $\operatorname{dim}(\operatorname{IDer} T)_{\sigma_{i}}=0$ while $\operatorname{dim}(\operatorname{IDer} T)_{\sigma_{1}+\sigma_{2}}=1$ (see [27, Lamma 2.4]). Or, if $I$ is maximal, i.e., $I$ represents all nonzero classes in $\mathbb{Z}^{n} /\left(2 \mathbb{Z}^{m} \oplus \mathbb{Z}^{n}\right) \cong \mathbb{Z}_{2}^{m}$, we get $\operatorname{dim}_{F}(\operatorname{IDer} T)_{\alpha}=2^{m-1}-1$ for all $\alpha \in \mathbb{Z}^{n} \backslash H$.

It remains to consider the Albert torus. Our analysis will be based on the following general lemma.

4.14. Lemma. Let $G=\mathbb{Z}_{3} \oplus \cdots \oplus \mathbb{Z}_{3}$ with $r \geq 1$ factors and let $J=\bigoplus_{g \in G} J_{g}$ be a Jordan $G$-torus over $F$ which is of strong type. For an $F$-vector space $V$ we consider an F-bilinear map $\{\cdot, \cdot\}: J \times J \rightarrow V$ satisfying

(i) $\{x y, z\}+\{y z, x\}+\{z x, y\}=0$

and

(ii) $\{x, y\}+\{y, x\}=0$. 
For $g \in G$ denote $\{J, J\}_{g}=\sum_{g=e+f}\left\{J_{e}, J_{f}\right\}$. Then

$$
\operatorname{dim}_{F}\{J, J\}_{g} \leq r-1 \text { for } g \neq 0 .
$$

Proof. We choose $0 \neq t_{g} \in J_{g}$ and note that then $t_{g} t_{h}=s t_{g+h}$ for some $0 \neq s \in F$. It will be convenient to use the abbreviation $\{x: y\}$ in case $\{x: y\}=s\{x, y\}$ for some $0 \neq s \in F$. Then

$$
\left\{t_{g}^{n}, t_{h}\right\}=n\left\{t_{g}: t_{(n-1) g+h}\right\} \quad \text { for } 0 \leq n \leq 2 .
$$

Indeed, $\{1, J\}=0$ by (i) and for $n=2$ we have $\left\{t_{g}^{2}, t_{h}\right\}=2\left\{t_{g}, t_{g} t_{h}\right\}=2\left\{t_{g}: t_{g+h}\right\}$. Next, we let $\varepsilon_{i}=0 \oplus \cdots \oplus 1 \oplus \cdots \oplus 0$ with $1 \in \mathbb{Z}_{3}$ at the $i$ th position and put $t_{i}:=t_{\varepsilon_{i}}$. We claim that for $g=e+f, e=\sum_{i} e_{i} \varepsilon_{i}$ with $0 \leq e_{i}<3$ we have

$$
\left\{t_{e}, t_{f}\right\}=\sum_{i=1}^{r}\left\{t_{i}^{e_{i}}: t_{g-e_{i} \varepsilon_{i}}\right\} .
$$

To prove (4.14.3) we observe that in general by (i), (ii) and commutativity of $J$, we have $\{x y, z\}=\{x, y z\}+\{y, x z\}$. Hence

$$
\begin{aligned}
\left\{t_{e}, t_{f}\right\} & =\left\{t_{1}^{e_{1}}\left(t_{2}^{e_{2}}\left(\cdots t_{r}^{e_{r}}\right) \cdots\right): t_{r}\right\} \\
& =\left\{t_{1}^{e_{1}}:\left(t_{2}^{e_{2}}\left(\cdots t_{r}^{e_{r}}\right) \cdots\right) t_{r}\right\}+\left\{\left(t_{2}^{e_{2}}\left(\cdots t_{r}^{e_{r}}\right) \cdots\right): t_{1}^{e_{1}} t_{r}\right\} \\
& =\left\{t_{1}^{e_{1}}: t_{e-e_{1} \varepsilon_{1}}\right\}+\left\{\left(t_{2}^{e_{2}}\left(\cdots t_{r}^{e_{r}}\right) \cdots\right): t_{e-e_{1} \varepsilon_{1}}\right\} .
\end{aligned}
$$

Continuing similarly with the second term proves (4.14.3). From (4.14.3) and (4.14.2) we then obtain

$$
\left\{t_{e}, t_{f}\right\}=\sum_{i=1}^{r} e_{i}\left\{t_{i}: t_{g-\varepsilon_{i}}\right\} .
$$

Now consider $g=\sum_{i=1}^{r} g_{i} \varepsilon_{i} \neq 0$. Applying (4.14.4) yields

$$
\begin{aligned}
\left\{t_{1}, t_{g-\varepsilon_{1}}\right\} & =-\left\{t_{g-\varepsilon_{1}}, t_{1}\right\} \\
& =-\left(g_{1}-1\right)\left\{t_{1}: t_{g-\varepsilon_{1}}\right\}-g_{2}\left\{t_{2}: t_{g-\varepsilon_{2}}\right\}-g_{3}\left\{t_{3}: t_{g-\varepsilon_{3}}\right\}-\cdots,
\end{aligned}
$$

whence $0=\sum_{i=1}^{r} g_{i}\left\{t_{i}: t_{g-\varepsilon_{i}}\right\}$, which gives a nontrivial relation among the $\left\{t_{i}, t_{g-\varepsilon_{i}}\right\}, i=1, \ldots, r$.

4.15. Theorem. For the Albert torus $\mathbb{A}_{t}$ we have

$$
\operatorname{dim}_{F}\left(\operatorname{IDer} \mathbb{A}_{t}\right)_{\alpha}=2 \text { for all } \alpha \in \mathbb{Z}^{n} \backslash H .
$$

Proof. We apply Lemma 4.14 to the central closure $J=\overline{\mathbb{A}}_{t}$ of $\mathbb{A}_{t}$. By 31 we know that $J=\bigoplus_{\bar{\alpha} \in \mathbb{Z}_{3}^{3}} J_{\bar{\alpha}}$ is a $\mathbb{Z}_{3}^{3}$-torus with $J_{\bar{\alpha}}=\bar{Z} \otimes_{Z} Z\left(\mathbb{A}_{t}\right)_{\alpha}$. Also, we let $\{x, y\}:=$ $\left[L_{x}, L_{y}\right]$ for $x, y \in J$. Since $\{J, J\}_{\bar{\alpha}}=(\operatorname{IDer} J)_{\bar{\alpha}}$, we get $\operatorname{dim}_{\bar{Z}}(\operatorname{IDer} J)_{\bar{\alpha}} \leq 2$ for $\bar{\alpha} \neq 0$ by Lemma 4.14 while (IDer $J)_{\overline{0}}=Z \otimes\left(\text { IDer } \mathbb{A}_{t}\right)_{H}=0$ by (1.4.2). On the other side, one knows that $\operatorname{Der}_{\bar{Z}} J=$ IDer $J$ is a simple Lie algebra of type $F_{4}$ (see [15, p. 21]), and so $\operatorname{dim}_{\bar{Z}}$ Der $J=52$. Hence

$$
52=\operatorname{dim}_{\bar{Z}} \operatorname{IDer} J=\sum_{\bar{\alpha} \neq 0} \operatorname{dim}_{\bar{Z}}(\operatorname{IDer} J)_{\bar{\alpha}} \leq 26 \cdot 2=52,
$$

and so $\operatorname{dim}_{F}\left(\text { IDer } \mathbb{A}_{t}\right)_{\alpha}=\operatorname{dim}_{\bar{Z}}(\text { IDer } J)_{\bar{\alpha}}=2$. 


\section{REFERENCES}

1. B. Allison, S. Azam, S. Berman, Y. Gao, and A. Pianzola, Extended affine Lie algebras and their root systems, Memoirs Amer. Math. Soc., vol. 603, Amer. Math. Soc., Providence, RI, 1997. MR 97i: 17015

2. Bruce N. Allison and Yun Gao, The root system and the core of an extended affine Lie algebra, Selecta Math. (N.S.) 7 (2001), no. 2, 149-212. MR 2002g:17041

3. G. Benkart, Derivations and invariant forms of Lie algebras graded by finite root systems, Canad. J. Math. 50 (1998), 225-241. MR 99d:17013

4. S. Berman, Y. Gao, and Y. Krylyuk, Quantum tori and the structure of elliptic quasi-simple Lie algebras, J. Funct. Anal. 135 (1996), 339-389. MR 97b:17007

5. S. Berman, Y. Gao, Y. Krylyuk, and E. Neher, The alternative torus and the structure of elliptic quasi-simple Lie algebras of type $A_{2}$, Trans. Amer. Math. Soc. 347 (1995), 4315-4363. MR 96b:17009

6. M. Boulagouaz, The graded and tame extensions, Lecture Notes in Pure and Appl. Math., Commutative Ring Theory, vol. 153, Marcel Dekker, New York, 1994. MR 94k:12010

7. D. Z. Doković and K. Zhao, Derivations, isomorphisms, and second cohomology of generalized Witt algebras, Trans. Amer. Math. Soc. 350(2) (1998), 643-664. MR 98d:17031

8. A. Duff, Derivations, invariant forms and central extensions of orthosymplectic Lie superalgebras, Ph.D. dissertation, University of Ottawa, Department of Mathematics and Statistics, 2002.

9. A. Dzhumadil'daev, Cohomologies and deformations of right-symmetric algebras, J. Math. Sci. (New York) 93 (1999), 836-876. MR 2000e:17002

10. _ Minimal identities for right-symmetric algebras, J. Algebra 225 (2000), 201-230. MR 2001b:17002

11. R. Farnsteiner, Derivations and central extensions of finitely generated graded Lie algebras, J. Algebra 118 (1988), 33-45. MR 89h:17018

12. K. R. Goodearl and E. S. Letzter, Quantum n-space as a quotient of classical n-space, Trans. Amer. Math. Soc. 352 (2000), 5855-5876. MR 2001e:16061

13. I. N. Herstein, Topics in ring theory, Chicago Lectures in Mathematics, The University of Chicago Press, 1969. MR 42:6018

14. N. Jacobson, Structure and representations of Jordan algebras, Amer. Math. Soc. Colloq. Publ., vol. 39, American Mathematical Society, Providence, RI, 1968. MR 40:4330

15. _ Exceptional Lie algebras, Lecture Notes in Pure and Applied Mathematics, Marcel Dekker, New York, 1971. MR 44:1707

16. N. Kawamoto, Generalizations of Witt algebras over a field of characteristic zero, Hiroshima Math. J. 16 (1986), 417-426. MR 88d:17017

17. M. Koecher, Imbedding of Jordan algebras into Lie algebras I, Amer. J. Math. 89 (1967), 787-816. MR 35:5480

18. T. Y. Lam, A first course in noncommutative rings, Graduate Texts in Mathematics, vol. 131, Springer-Verlag, New York, 1991. MR 92f:16001

19. O. Loos, Spiegelungsräume und homogene symmetrische Räume, Math. Z. 99 (1967), 141170. MR 35:3608

20. K. McCrimmon, Alternative algebras, (unpublished book).

21. Z_ Zelmanov's prime theorem for quadratic Jordan algebras, J. Algebra 76 (1982), 297326. MR 83h:17019

22. - Jordan centroids, Comm. Algebra 27, no. 2 (1999), 933-954. MR 2000b:17041

23. C. Năstăsescu and F. van Oystaeyen, Graded ring theory, North-Holland Mathematical Library, vol. 28, North-Holland, 1982. MR 84i:16002

24. J. M. Osborn and D. S. Passman, Derivations of skew polynomial rings, J. Algebra 176 (1995), 417-448. MR 97i:16030

25. D. S. Passman, Infinite crossed products, Pure and Applied Mathematics, vol. 135, Academic Press, Boston, 1989. MR 90g:16002

26. _ Simple Lie algebras of Witt type, J. Algebra 206 (1998), 682-692. MR 99j:17012

27. S. Tan, TTK algebras and vertex operator representations, J. Algebra 211 (1999), 298-342. MR 2000f:17035

28. X. Xu, New generalized simple Lie algebras of Cartan type over a field with characteristic zero, J. Algebra 224 (2000), 23-58. MR 2001b:17021 
29. Y. Yoshii, Classification of division $\mathbf{Z}^{\mathbf{n}}$-graded alternative algebras, to appear in J. Algebra.

30. — Quantum tori with graded involution, to appear in Canad. Math. Bull.

31. Coordinate algebras of extended affine Lie algebras of type $A_{1}$, J. Algebra 234 (2000), 128-168. MR 2001i:17031

32. Root-graded Lie algebras with compatible grading, Comm. Algebra 29 (2001), no. 8, 3365-3391. MR 2002g:17044

33. K. A. Zhevlakov, A. M. Slinko, J. P. Shestakov, and A. I. Shirshov, Rings that are nearly associative, Academic Press, New York, 1982. MR 83i:17001

Department of Mathematics and Statistics, University of Ottawa, Ottawa, Ontario K1N 6N5, CANADA

E-mail address: neher@uottawa.ca

Department of Mathematical and Statistical Sciences, University of Alberta, Edmonton, Alberta T6G 2G1, CAnada

E-mail address: yoshii@math.ualberta.ca

Current address: Department of Mathematics, Van Vleck Hall, University of WisconsinMadison, Madison, Wisconsin 53706

E-mail address: yoshii@math.wisc.edu 